$1 / .29888(1)$

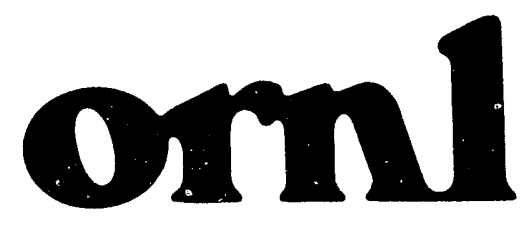

ORNL/TM-12003

\section{OAK RIDGE NATIONAL LABORATORY}

MARTIN MAVIETTA

\section{A Parallel Algorithm for the Non-Symmetric Eigenvalue Problem}

\author{
Jack Dongarra \\ Majed Sidani
}

MANAGED BY

MARTIN MARIETTA ENERGY SYSTEMS, INC.

FOR THE UNITED STATES

DEPARTMENT OF ENERGY 
This report has been reproduced directly from the best available copy.

Available to DOE and DOE contractors from the Office of Scientific and Technical Information, P.O. Box 62, Oak Ridge, TN 37831; prices available from (615) 576-8401. FTS 626-8401.

Available to the public from the National Technical Information Service, U.S. Department of Commerce, 5285 Port Royal Rd., Springfield, VA 22161.

NTIS price codes--Printed Copy: A07 Microfiche AO1

This report was prepared as an account of work sponsored by an agency of the United States Government. Neither the United States Government nor any agency thereof, nor any of their employees, makes any warranty, express or implied, or assumes any legal liability or responsibility for the accuracy, completeness, or usefulness of any information, apparatus, product, or process disclosed, or represents that its use would not infringe privately owned rights. Reference herein to any specific commercial product, process, or service by trade name, trademark, manufacturer, or otherwise, does not necessarily constitute or imply its endorsement, recommendation, or favoring by the United States Government or any agency thereof. The views and opinions of authors expressed herein do not necessarily state or reflect those of the United States Government or any agency thereof. 


\title{
A PARALLEL ALGORITHM FOR THE NON-SYMMETRIC EIGENVALUE PROBLEM
}

\author{
Jack Dongarra \\ Majed Sidani \\ Computer Science Department \\ University of Tennessee \\ Knoxville, TN 37996 \\ and \\ Mathematical Sciences Section \\ Engineering Physics and Mathematics \\ P.O. Box 2008, Bldg. 6012 \\ Oak Ridge National Laboratory \\ Oak Ridge, TN 37831-6367 \\ DATE PUBLISHED - DECEMBER 1991 \\ This work was supported in part by the Naticnal Science Foundation \\ Science and Technology Center Cooperative Agreement No. CCR-8809615, and \\ in part by the Applied Mathematical Sciences subprogram of the Office \\ of Energy Research, U.S. Department of Energy.
}

Prepared by the

Oak Ridge National Laboratory

Oak Ridge, Tennessee 37831

managed by

MARTIN MARIETTA ENERGY SYSTEMS, INC.

for the

U.S. DEPARTMENT OF ENERGY

under Contract No. DE-ACO5-840R21400 


\section{CONTENTS}

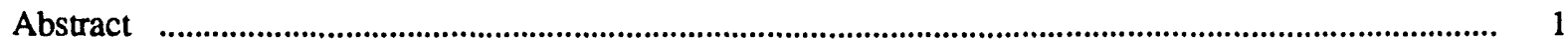

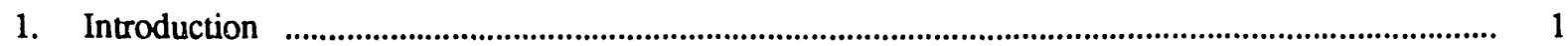

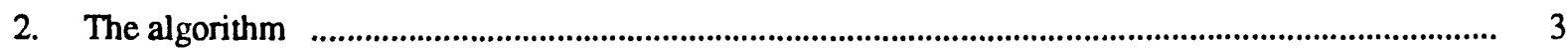

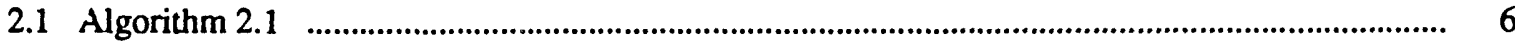

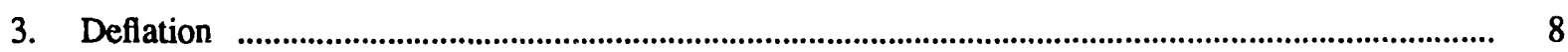

3.1 Deflation using elementary transformations ......................................................................... 9

3.2 Deflation with help from the left eigenvector ............................................................................... 14

3.3 Deflation with orthogonal transformations ................................................................................. 16

3.4 Remarks on identifying duplicate eigenvalues .................................................................... 19

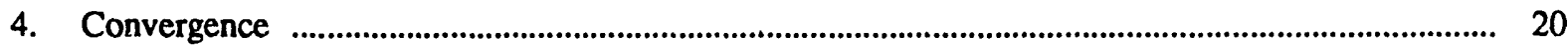

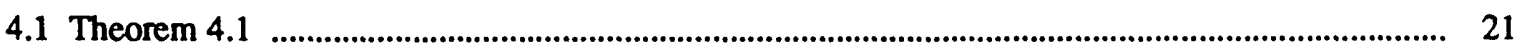

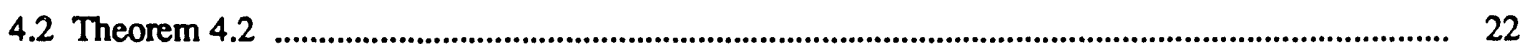

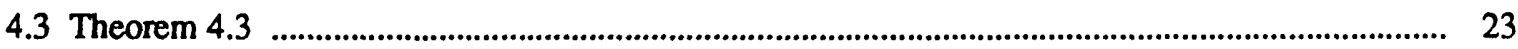

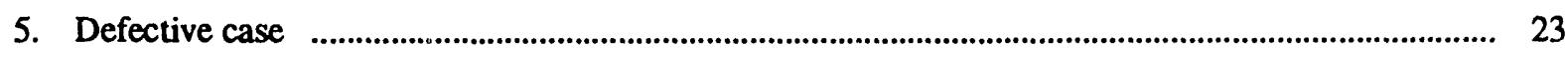

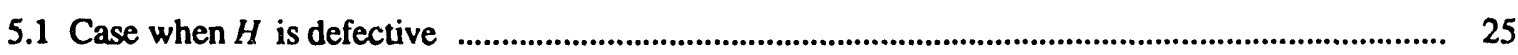

5.2 Case when $H_{0}$ is defective

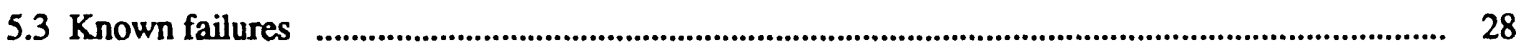

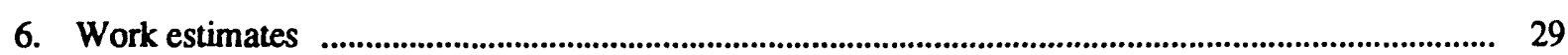

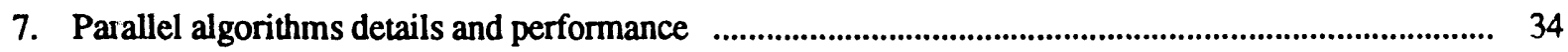

7.1 Shared memory implementation ........................................................................................... 34

7.2 Distributed memory implementation ................................................................................... 35

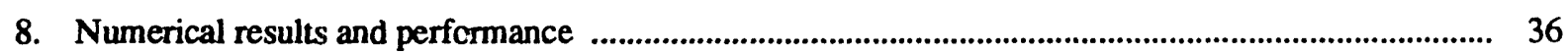

9. The generalized eigenvalue problem .............................................................................................. 38

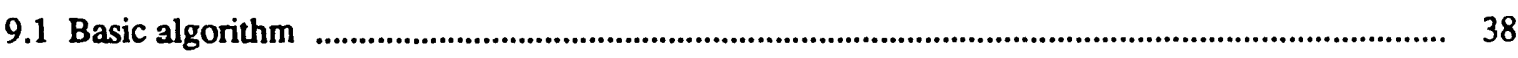

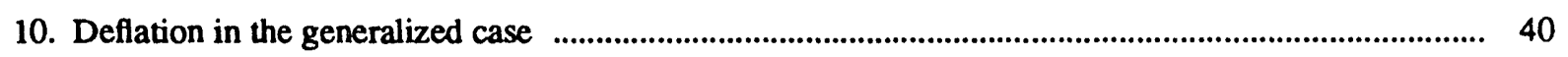

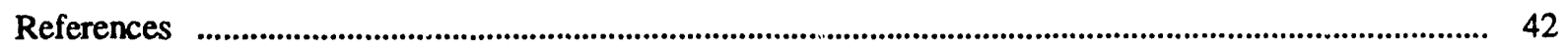




\title{
A Parallel Algorithm for the Non-Symmetric Eigenvalue Problem *
}

\author{
Jack J. Dongarra \\ and \\ Majed Sidani \\ Computer Science Department \\ University of Tennessee \\ Knoxville, TN 37996-1301 \\ and \\ Mathematical Sciences Section \\ Oak Ridge National Laboratory \\ Oak Ridge, TN 37831
}

August 7, 1991

\begin{abstract}
This paper describes a parallel algorithm for computing the eigenvalues and eigenvectors of a non-symmetric matrix. The algorithm is based on a divide-and-conquer procedure and uses an iterative refinement technique.
\end{abstract}

\section{Introduction}

The algebraic eigenvalue problem is one of the fundamental problems in computational mathematics. It arises in many applications and therefore represents an important area of algorithmic research. The problem has received considerable attention which has resulted in reliable methods $[16,15,14]$. However it is reasonable to expect that calculations might be accelerated through the use of parallel algorithms. A fully parallel algorithm for the symmetric eigenvalue problem was recently proposed in [6]. This algorithm is based on a divide-and-conquer procedure outlined in [3]. The latter was based on work by [9] and [1]. The fundamental principle behind this algorithm is that the partitioning by rank-one tearing interlaces the eigenvalues of the modified problem with the eigenvalues of the original problem. This approach in turn enables rapid and

-This work was supported in part by the National Science Foundation Science and Technology Center Cooperative Agreement No. CCR-8809615, and in part by the Ápplied Miathematicai Sciences subprogram of the Office of Energy Research, U.S Department of Energy, under Contract DE-AC05-84OR21400. 
accurate determination, in parallel, of the eigenvalues and subsequent eigenvectors.

In this paper we propose a parallel algorithm for the solution of the non-symmetric eigenvalue problem. The approach uses some of the features of the divide-and-conquer algorithm for the symmetric case mentioned earlier. In particular, the original problem is divided into two smaller and independent subproblems by a rank-one modification of the matrix. (We assume that the matrix has already been reduced to Hessenberg form and that the rank-one modification removes a subdiagonal element). Once the eigensystems of the smaller subproblems are known, it is possible to compute those of the original matrix. In the non-symmetric case, however, the eigenvalues of the modified matrix do not interlace with those of the original matrix. Indeed, the eigenvalues can scatter anywhere in the complex plane.

In our algorithm for the non-symmetric case, the eigensystem of the subproblem is used only to construct initial guesses for an iterative process that yields the desired eigensystem of the original problem. Under suitable conditions, Newton's method or a continuation method can be used to find the eigenpairs of the original problem. We report here on our application of an iterative refinement approach based on Newton's method; we shall not pursue the continuation method in this paper. Work on the continuation approach has been reported by [12].

In section 2 we describe the algorithm using an iterative refinement procedure based on Newton's met? od. Section 3 covers the deflation step required to overcome multiple convergence to a particular eigenvalue. In section 4 the convergence behavior is discussed. In section 5 we discuss the case when the matrix or its rank one modification has a defective system of eigenvectors. Section 6 estimates the amount of work the parallel algorithm requires and compares this to the standard techniques. Section 7 describes the parallel algorithm and the different parallel implementations of the new algorithm, and gives numerical results. Section 9 describes how our ideas extend to the generalized eigenvalue problem. 


\section{The Algorithm}

Given a matrix $H$, an eigenpair $\left(x_{0}, \lambda_{0}\right)$ of $H$ can be thought of as a solution to the following polynomial system

$$
\text { (S) }\left\{\begin{array}{l}
H x-\lambda x=0 \\
L(x)=1
\end{array}\right.
$$

where $L(x)$ is a scalar equation. Here, we set $L(x)=e_{s}^{T} x$, where $e_{s}$ is the $s^{\text {th }}$ unit vector. Let

$$
F_{s}(x, \lambda)=\left(\begin{array}{c}
H x-\lambda x \\
e_{s}^{T} x-1
\end{array}\right) .
$$

Then, finding an eigenpair of $H$ reduces to finding a zero of $F_{s}$. In what follows, unless otherwise mentioned, $H$ is assumed to be a real, unreduced (no zeros on the subdiagonal), upperHessenberg matrix of order $n$. This is no restriction on the type of problems we want to solve, since if $H$ has a zero on the subdiagonal then, finding its eigenvalues reduces to finding those of the blocks on the diagonal. We note also that as a consequence of our assumption that $H$ is unreduced, an eigenvalue of $H$ can only have geometric multiplicity one: this is quite easy to see since the first $n-1$ columns of $H-\lambda I$ are linearly independent. We will assume for now that $H$ has a simple spectrum. We can write $H$ as

$$
H=\left(\begin{array}{c|c}
H_{11} & H_{12} \\
\hline \alpha e_{1}^{(k)} e_{k}^{(k) T} & H_{22}
\end{array}\right),
$$

where $H_{11}$ and $H_{22}$ are upper-Hessenberg of dimensions $k \times k$ and $n-k \times n-k$, respectively; $\alpha=h_{k+1, k}$, and $e_{i}^{(k)}$ is the $i^{\text {th }}$ unit vector of length $k$.

Let $H_{0}=H-\alpha e_{k+1}^{(n)} e_{k}^{(n) T}$, then $H_{0}=\left(\begin{array}{c|c}H_{11} & H_{12} \\ \hline 0 & H_{22}\end{array}\right)$, and $\sigma\left(H_{0}\right)=\sigma\left(H_{11}\right) \cup \sigma\left(H_{22}\right)$ (where $\sigma(M)$ is the spectrum of $M)$. The algorithm can then be described as follows: We first find the $k$ eigenpairs of $H_{11}$ and the $n-k$ eigenpairs of $H_{22}$ by some method, perhaps the QR algorithm. These eigenpairs are then used to construct initial approximations to the eigenpairs of $H$ in the following way: if $\lambda$ is an eigenvalue of $H_{11}$ and $x$ is the corresponding eigenvector, then $\lambda$ is viewed as an approximate eigenvalue of $H$ with the corresponding approximate eigenvector taken to be $\left(\begin{array}{l}x \\ 0\end{array}\right)$, where $n-k$ zeros are appended to $x$. Note that $\left(\begin{array}{l}x \\ 0\end{array}\right)$ is an exact eigenvector of 
$H_{0}$ corresponding to $\lambda$. On the other hand, if $\lambda$ is an eigenvalue of $H_{22}$ and $x$ is the corresponding eigenvector, then $\left(\begin{array}{l}0 \\ x\end{array}\right)$, where $k$ zeros are prefixed to $x$ is taken as an approximate eigenvector of $H$ corresponding to the approximate eigenvalue $\lambda$. If $\lambda$ is not an eigenvalue of $H_{11}$ then the last $n-k$ components of $\left(\begin{array}{l}0 \\ x\end{array}\right)$, i.e., those of $x$, are the trailing components of an exact eigenvector of $H_{0}$ corresponding to $\lambda$, namely: $\left(\begin{array}{c}\left(H_{11}-\lambda\right)^{-1} H_{12} x \\ x\end{array}\right)$. However, if $\lambda$ is an eigenvalue of $H_{11}$, and hence a necessarily multiple eigenvalue of $H_{0}$, then if $\lambda$ has geometric multiplicity one, no eigenvector of $H_{0}$ will have the components of $x$ as its trailing components.

Having constructed these initial guesses, we now use them as starting points for Newton's method to find the zeros of $F_{s}$, i.e., the eigenpairs of $H$. Hence, given an approximate zero $(x, \lambda)$ of $F_{s}$ the next approximation is

$$
x^{\prime}=x+y ; \lambda^{\prime}=\lambda+\mu,
$$

where $(y, \mu)$ is the solution of the system

$$
\left(\begin{array}{cc}
H-\lambda I & -x \\
e_{s}^{T} & 0
\end{array}\right)\left(\begin{array}{c}
y \\
\mu
\end{array}\right)=\left(\begin{array}{c}
r \\
0
\end{array}\right),
$$

where $r=\lambda x-H x$.

Newton's method comes into this problem in a rather "natural" way. Indeed, suppose that $(x, \lambda)$ is an approximate eigenpair of $H$,

$$
H x \approx \lambda x,
$$

and let us find a way to compute a correction $(y, \mu)$ to this approximate eigenpair. Clearly, $(y, \mu)$ should satisfy

$$
H(x+y)=(\lambda+\mu)(x+y)
$$

Rearranging the latter equation yields

$$
(H-\lambda) y-\mu x=\lambda x-H x+\mu y .
$$

Now if we ignore the second order term $\mu y$, and if we impose a normalization condition on $x$, say $x_{s}=1$ and assume that the desired eigenvector should satisfy the same condition, then $(y, \mu)$ is 
the solution of

$$
\left(\begin{array}{cc}
H-\lambda I & -x \\
e_{s}^{T} & 0
\end{array}\right)\left(\begin{array}{l}
y \\
\mu
\end{array}\right)=\left(\begin{array}{l}
r \\
0
\end{array}\right),
$$

with $r$ as above. But, this is the same equation that we obtain when a Newton iteration is applied to the function $\vec{F}_{s}$ to find a correction to $(x, \lambda)$, as we can see from $(1)$. We note here a result from [4], where the author studied an iterative refinement technique to compute the correction $(y, \mu)$ to $(x, \lambda)$ from equation (2) (i.e., without ignoring the second order term) and proved that in exact arithmetic, that method and Newton's method produce the same final iteration.

So far we have not attempted to answer the question of which subdiagonal entry to introduce the zero. We will use first order perturbation theory in order to shed some light on this issue. Let $E=-\alpha e_{k+1} e_{k}^{T}$, where as above: $\alpha=h_{k+1, k}$ (note that $H+E=H_{0}$, introduced above). Then classical results from function theory ([11], v. 2, pp. 119-134) allow us to state that in a small neighborhood of zero, we have

$$
(H+\epsilon E) x(\epsilon)=\lambda(\epsilon) x(\epsilon)
$$

for all $\epsilon$ in that neighborhood and for differentiable $(x(\epsilon), \lambda(\epsilon))$ corresponding to a simple eigenpair $(x, \lambda)$ of $H$. Clearly: $x(0)=x$ and $\lambda(0)=\lambda$. Let $y^{H}$ be the left eigenvector of $H$ corresponding to $\lambda$. Then differentiating both sides we have (with primes indicating derivatives),

$$
H x^{\prime}(\epsilon)+E x(\epsilon)+\epsilon E x^{\prime}(\epsilon)=\lambda^{\prime}(\epsilon) x(\epsilon)+\lambda(\epsilon) x^{\prime}(\epsilon)
$$

Multiplying by $y^{H}$ and setting $\epsilon=0$ we get

$$
y^{H} E x=\lambda^{\prime}(0) y^{H} x
$$

and therefore

$$
\left|\lambda^{\prime}(0)\right|=\frac{\left|y^{H} E x\right|}{\left|y^{H} x\right|}=\frac{|\alpha|\left|y_{k+1}\right|\left|x_{k}\right|}{\left|y^{H} x\right|} .
$$

The quantities that vary with $k$ in this expression for the rate of change of $\lambda$, are the factors in the numerator. However, $|\alpha|,\left|y_{k+1}\right|$ and $\left|x_{k}\right|$ are not really independent of one another: indeed, 
if $\alpha=0$, then at least one of $y_{k+1}$ or $x_{k}$ is zero. Hence for $\alpha$ small, we can expect one of $y_{k+1}$ and $x_{k}$ to be correspondingly small, since the components of the eigenvectors vary continuously. Therefore, we have found it sufficient in practice to look for the smallest subdiagonal entry in a pre-specified range, and accept it as the subdiagonal entry (in that range) with respect to which the eigenvalues of the matrix are least sensitive, and set it equal to zero.

An outline of the algorithm follows:

Algorithm 2.1 Given an upper-Hessenberg matrix $H$, the following algorithm computes the eigensystems of two submatrices of $H$ and uses them as initial guesses for starting Newton iterations for determining the eigensystem of $H$.

Determine subdiagonal element $\alpha$ where $H=\left(\begin{array}{c|c}H_{11} & H_{12} \\ \hline 0^{\alpha} & H_{22}\end{array}\right)$, should be split;

Determine initial guesses from eigensystems of the 2 diagonal blocks $H_{11}$ and $H_{22}$;

For each initial guess $\left(\lambda_{i}, \tau_{i}\right)$ iterate until convergence:

$$
\left(\begin{array}{cc}
H-\lambda_{i} I & -x_{i} \\
e_{0}^{T} & 0
\end{array}\right)\left(\begin{array}{l}
y \\
\mu
\end{array}\right)=\left(\begin{array}{c}
r_{i} \\
0
\end{array}\right) ; \lambda_{i}-\lambda_{i}+\mu ; x_{i}-x_{i}+y
$$

end;

Check for duplicates and deflate if necessary;

More will be said about the last step, deflation, in section 3. The algorithm just described is inherently parallel. Indeed, the eigensystems of $H_{11}$ and $H_{22}$ can be computed in parallel. After which Newton's method can be started with different initial guesses on different processors. Furthermore, the dividing process can be applied recursively to obtain yet smaller subproblems, i.e., $H_{11}$ and $H_{22}$ can be divided each into subproblems. This will permit the use of more processors. However, care must be exercised if the algorithm is to be kept efficient. More will be said about this in section 6 (work estimate). In practice, the original matrix will be dense 
and we will need to reduce it to upper-Hessenberg form as a first step; this can be done in a stable fashion through a sequerice of orthogonal similarity transformations.

A brief study ci some sufficient conditions guaranteeing the convergence of our method will be touched upon in section 4 . Now assuming that the algorithm converges, it could happen that the same eigenpair of $H$ is obtained more than once: i.e.,starting from two (or more) distinct initial approximations Newton's method converges to the same eigenpair of $H$. We have investigated methods to obtain further eigenpairs of $H$ should this happen (see section 3 ).

We end this section with some implementational details. Our algorithm will accept $(x, \lambda)$ as an eigenpair of $H$ when $\|H x-\lambda x\| /\|x\|\|H\|<t o l$, where tol is sorne specified tolerance of order $\epsilon$, the machine unit roundoff. Under these conditions $([10]),(x, \lambda)$ is an exact eigenpair of a matrix obtained from $H$ by a slight perturbation: Indeed,

$$
\left(H+\frac{1}{x^{H} x} r x^{H}\right) x=\lambda x
$$

where $r=\lambda x-H x$.

Starting from two complex conjugate initial approximations, Newton's method will converge to two complex conjugate zeros of $F_{s}$, or the same real zero. To prove this it suffices to look at one iteration and show that when the current approximations are complex conjugate, Newton's method yields two complex conjugate corrections, or the same real correction. The system we have to solve starting from $(x, \lambda)$ is $(1)$. For $(\bar{x}, \bar{\lambda})$ this becomes

$$
\left(\begin{array}{cc}
H-\bar{\lambda} I & -\bar{x} \\
e_{s}^{T} & 0
\end{array}\right)\left(\begin{array}{c}
y^{\prime} \\
\mu^{\prime}
\end{array}\right)=\left(\begin{array}{c}
\bar{r} \\
0
\end{array}\right) .
$$

But this is the same linear system obtained when the conjugate of both sides in (1) is taken, knowing that the matrix is real and hence $\bar{H}=H$. Therefore: $y^{\prime}=\bar{y}$ and $\mu^{\prime}=\bar{\mu}$. This will allow significant savings in the computations.

Lastly when starting from a real initial guess, only real corrections are computed. Therefore the imaginary part of the eigenvalue should be perturbed if convergence to a complex eigenpair 
is to be made possible. In practice we have done this when real arithmetic does not lead to convergence after a pre-specified number of corrections. To illustrate the situation when perturbing the imaginary part of the eigenvalue is necessary we mention the very simple case of a $2 \times 2$ real matrix whose eigenvalues are complex.

\section{Deflation}

When Newton's method is applied to solve the eigensystem of a matrix $\mathrm{H}$, it is possible that starting from distinct initial guesses we converge to the same eigenpair of $H$. In fact a naive implementation of the algorithm in section 2 may result in many eigenvalues being found multiple times and consequently some eigenvalues not being found at all since the number of initial guesses is $n$ (at most). To avoid this unwanted situation, we included a deflation step in our algorithm which is designed to obtain further zeros using Newton's method. In this section we prop ise various methods for doing this. Basically, these methods fall into twr classes.

Assume that when algorithm 2.1 is applied to the $n \times n$ matrix $! I$ (we will assume for simplicity that it has no multiple eigenvalues) the eigenpair $(x, \lambda)$ of $H$ is obtained more than once, i.e., the algorithm converges to $(x, \lambda)$ from several distinct initial guesses $\left(x_{0}^{(i)}, \lambda_{0}^{(i)}\right), i=1, \ldots, r, r>1$. Then the methods of one class produce a $n-1 \times n-1$ matrix $H^{\prime}$ such that $\sigma\left(H^{\prime}\right)=\sigma(H)-\{\lambda\}$ and apply algorithm 2.1 to $H^{\prime}$ starting from $r-1$ of these initial guesses; indeed, we can expect $r-1$ eigenpairs of $H$ to have been missed. If the algorithm converges, then it will do so to eigenpairs different from $(x, \lambda)$ since $\lambda$ is no longer in the spectrum.

The $n$ thei class of methods will reapply algorithm 2.1 to the original matrix $H$ starting from $r-1$ of the initial guesses mentioned above, but will force convergence away from $(x, \lambda)$ by ensuring at all steps that the current eigenvector forms a non zero angle with $x$.

A common drawback that these methods have is that they tend to serialize the computation. However, it has been our experience that the need to deflate arises infrequently, less than $5 \%$ of 
the time in our tests.

\subsection{Deflation using elementary transformations}

We now describe one possible deflating similarity transformation. The assumptions on $H, \lambda$ and $x$ are as above.

Since we are assuming $H$ to be upper-Hessenberg with no zeros on the subdiagonal, then $x_{n} \neq 0$ and the elementary transformation

$$
M=\left[e_{1}, \ldots, e_{n-1}, x\right]
$$

i.e.,

$$
M=\left(\begin{array}{cccc}
1 & & & x_{1} \\
& \ddots & & \vdots \\
& & 1 & x_{n-1} \\
0 & & & x_{n}
\end{array}\right),
$$

is non-singular. The inverse of this matrix is

$$
M^{-1}=\left(\begin{array}{cccc}
1 & & & -\frac{x_{1}}{x_{n}} \\
& \ddots & & \vdots \\
& & 1 & -\frac{\dot{x}_{n-1}}{x_{n}} \\
0 & & & \frac{1}{x_{n}}
\end{array}\right) .
$$

It is easy to see that $M^{-1} x=e_{n}$. Now we let

$$
\widetilde{H}=M^{-1} H M \text {. }
$$

Then it is easy to.verify that the last column of $\widetilde{H}$ is $\lambda e_{n}$. Furthermore the leading principal submatrix of order $n-1$ of $\widetilde{H}$, which we will call $H^{\prime}$, is upper Hess oerg, has the property that

$$
\sigma\left(H^{\prime}\right)=\sigma(H)-\{\lambda\}
$$

and differs from the leading $n-1 \times n-1$ principal submatrix of $H$ in the last column only. In fact, if we let $h_{n-1}$ be the iast column of the leading principal submatrix of $H$ of order $n-1$, then it is straightforward to verify that the last column of $H^{\prime}$ is $h_{n-1}-h_{n, n-1} x^{\prime}$, where

$$
x^{i}=\left(\begin{array}{c}
\frac{x_{1}}{x_{n}} \\
\vdots \\
\frac{x_{n-1}}{x_{n}}
\end{array}\right) \text {. }
$$


The strategy we have just described is given in [16] and applies regardless of whether the eigenpair $(x, \lambda)$ is real or not. However, when $(x, \lambda)$ is real we get a real matrix $H^{\prime}$. In the case when $(x, \lambda)$ is not real, the last column of $H^{\prime}$ alone is not real since, as we remarked earlier, the other columns are those of a leading principal submatrix of $H$. Hence the leading principal submatrix of $H^{\prime}$ of order $n-2$ is real. Also in this case the complex conjugate of $(x, \lambda),(\bar{x}, \bar{\lambda})$, is an eigenpair of $H$, and therefore $\bar{\lambda}$ is an eigenvalue of $\tilde{H}$; a corresponding eigenvector is

$$
M^{-1} \bar{x}=\left(\begin{array}{c}
\bar{x}_{1}-x_{1} \frac{\bar{x}_{n}}{x_{n}} \\
\vdots \\
\bar{x}_{n-1}-x_{n-1} \frac{\underline{x}_{n}}{x_{n}} \\
\frac{x_{n}}{x_{n}}
\end{array}\right) .
$$

Since $\sigma\left(H^{\prime}\right)=\sigma(H)-\{\lambda\}, \bar{\lambda}$ is an eigenvalue of $H^{\prime}$ and a corresponding eigenvector is the vecior $\tilde{x}$ of length $n-1$, whose components are the first $n-1$ components of a scalar multiple of $M^{-1} \bar{x}$ :

$$
\tilde{x}=\left(\begin{array}{c}
\left(\bar{x}_{1} x_{n}-x_{1} \bar{x}_{n}\right) / i \\
\vdots \\
\left(\bar{x}_{n-1} x_{n}-x_{n-1} \bar{x}_{n}\right) / i
\end{array}\right),
$$

where $i^{2}=-1$. This is due to the special structure of $\tilde{H}$. Now recall that $H^{\prime}$ has no zeros on the subdiagonal, and so we know that the last component of $\tilde{x}$ is nonzero. Note that $\tilde{x}$ is real. We can carry out a deflation that produces a matrix $H^{\prime \prime}$ of order $n-2$ with the property that

$$
\sigma\left(H^{\prime \prime}\right)=\sigma\left(H^{\prime}\right)-\{\bar{\lambda}\}
$$

in the same way we obtained $H^{\prime}$ from $H$ using the elementary transformation of order $n-1$

$$
M^{\prime}=\left(\begin{array}{cccc}
1 & & & \tilde{x}_{1} \\
& & \ddots & \vdots \\
& 0 & & \tilde{x}_{n-2} \\
& 0
\end{array}\right) .
$$

By a previous remark about this deflation strategy, we know that $H^{\prime \prime}$ differs from the leading principal submatrix of $H^{\prime}$ of order $n-2$ (which is real) in the last column only. The last column of $H^{\prime \prime}$ is $h_{n-2}^{\prime}-h_{n-1, n-2} x^{\prime \prime}$, where $h_{n-2}^{\prime}$ is the last column of the leading principal submatrix of $H^{\prime}$ and $x^{\prime \prime}$ is the vector whose components are the first $n-2$ components of $\tilde{x}$. Since all of the quantities involved are real, $H^{\prime \prime}$ is real. 
We remark here, as can be readily realized, that $H^{\prime}$ (or $H^{\prime \prime}$ ) is quite cheap to obtain in practice once an eigenpair of $H$ is available. It requires $O(n)$ operations consisting of a vector normal. ization, a scalar-vector multiplication and a vector-vector addition. However the conditioning of the matrix $M$ might raise concern. Indeed:

$$
\operatorname{cond}_{\infty}(M)=\|M\|_{\infty}\left\|M^{-1}\right\|_{\infty} \approx \max \left(\left|x_{i}\right|\right) \max \left(\frac{\left|x_{i}\right|}{\left|x_{n}\right|}\right)
$$

which can be large if $x_{n} \ll x_{i}$. Having noted this, it is clear that the ill-conditioning of $M$ can be easily detected, and therefore one of the more stable (and costlier) methods which we introduce next and in the following sections can be used.

It is possible to prevent the ill-conditioning of $M$ from bearing on the algorithm by avoiding a similarity transformation. More precisely, the eigenvalue problem we want to solve can be thought of as a generalized eigenvalue problem,

$$
H x=\lambda B x,
$$

with $B=I$. We want to find $\sigma(H)=\sigma(H, I)$. Now we know that given any non-singular $M$ and $M^{\prime}$,

$$
\sigma(H, I)=\sigma\left(M^{\prime} H M, M^{\prime} M\right)
$$

Given a particular eigenpair $(x, \lambda)$ we would like to choose $M$ and $M^{\prime}$ in a way that solves the problem we set to ourselves at the beginning of this section, namely, we want to reduce the problem to one where $\lambda$ is no longer in the spectrum. A closer look at the similarity transformation introduced above, reveals that its deflating property is due to the fact that $M^{-1} x=e_{n}$. But then $M^{-1}$ is not the only matrix that can be used to accomplish this. In fact the following matrix $M^{\prime}$ can be chosen to reduce $x$ to a multiple of $e_{n}$ :

$$
M^{\prime}=D M^{-1}
$$

where $D$ is the diagonal matrix with the following entries

$$
\left\{\begin{array}{c}
d_{i}=1, \text { if } \frac{\left|x_{i}\right|}{\left|x_{n}\right|} \leq 1 \\
d_{i}=-\frac{x_{n}}{x_{i}}, \text { if } \frac{\left|x_{i}\right|}{\left|x_{n}\right|}>1
\end{array}\right.
$$


i.e., $D$ is chosen so that all the entries in $M^{\prime}$ are less than or equal to one. Then we have

$$
M^{\prime} H M=\left(\begin{array}{c|c}
H^{\prime} & 0 \\
\hline 0 & \gamma
\end{array}\right), M^{\prime} M=\left(\begin{array}{c|c}
D^{\prime} & 0 \\
\hline 0 & \delta
\end{array}\right)
$$

with

$$
\lambda=\gamma / \delta
$$

Also,

$$
\sigma\left(H^{\prime}, D^{\prime}\right)=\sigma(H, I)-\{\lambda\}
$$

and therefore by this transformation $\lambda$ has been "removed" from the spectrum. Working on the solution of a generalized eigenvalue problem from this point on will not in general cause any dramatic increase in the cost of the algorithm mainly because $D^{\prime}$ is diagonal. A Newton step with this problem involves the following computation

$$
\left(\begin{array}{cc}
H^{\prime}-\lambda_{i} D^{\prime} & -D^{\prime} x_{i} \\
e_{s}^{T} & 0
\end{array}\right)\left(\begin{array}{c}
y \\
\mu
\end{array}\right)=\left(\begin{array}{c}
r_{i} \\
0
\end{array}\right), \lambda_{i} \leftarrow \lambda_{i}+\mu ; x_{i} \leftarrow x_{i}+y
$$

where

$$
r_{i}=\lambda_{i} D^{\prime} x_{i}-H x_{i}
$$

Clearly the previous computation involves an $O(n)$ increase in the cost of one step: this comes from the multiplications by $D^{\prime}$. The details on how equation (8) is derived are given in section 9 . If $\lambda$ is complex, then after deflating $\lambda$ and its conjugate $\bar{\lambda}$ the resulting matrices $H^{\prime}$ and $D^{\prime}$ are complex in general. This is the major drawback of this method.

Finally we mention another approach that can be of interest when the similarity transformation (6) involves a very ill-conditioned $M(4)$. This approach consists of interchanging two components of $x$ and the corresponding columns and rows in $H$ so that the last component of $x$ is large enough. More precisely: Let $x_{s}$ be the largest component of $x$ (in absolute value) and let $P_{n s}$ be the matrix obtained from the identity matrix by perinuting the $n^{\text {th }}$ and $s^{\text {th }}$ columns. Then $\left(P_{n s} x, \lambda\right)$ is an eigenpair of the matrix $P_{n s} H P_{n s}$, since

$$
\left(P_{n s} H P_{n s}\right)\left(P_{n s} x\right)=\lambda P_{n s} x
$$


Now scale the vector $P_{n s} x$ so that the last component is 1 and call that vector $\tilde{x}$. Then, as above, the elementary transformation

$$
M=\left(\begin{array}{cccc}
1 & & \tilde{x}_{1} \\
& & \ddots & \vdots \\
& 0 & & \tilde{x}_{n-1} \\
& 0 & & 1
\end{array}\right)
$$

can be used to deflate the matrix $P_{n s} H P_{n s}$. The fact that here $P_{n s} H P_{n s}$ is not upper-Hessenberg is of no consequence. In fact, we can make the following general statement: Given any matrix $A$ of order $n$, and any eigenpair $(x, \lambda)$ of $A$, then a matrix $Q$ satisfying

$$
Q^{-1} x=e_{1} \text { or } Q^{-1} x=e_{n}
$$

can be used to deflate $A$, in the sense that

$$
Q^{-1} A Q=\left[\lambda e_{1}, B_{1}\right] \text { or } Q^{-1} A Q=\left[B_{2}, \lambda e_{n}\right]
$$

respectively; where $B_{1}$ and $B_{2}$ are $n \times(n-1)$ matrices.

Having thus deflated the matrix $P_{n s} H P_{n s}$, the leading principal submatrix of order $n-1$ of

$$
M^{-1} P_{n s} H P_{n s} M
$$

call it $H^{\prime}$, has all the eigenvalues of $H$ except $\lambda$ (if $\lambda$ is simple). However, $H^{\prime}$ is not upperHessenberg in general and therefore will be reduced back to Hessenberg form before applying Newton's iterations; this is meant to save on the cost of factorizing the Jacobian when solving the linear systems arising at each step of Newton's iteration. Note that it is only the trailing diagonal submatrix oi order $n-s+1 \times n-s+1$ of $H^{\prime}$ that needs to be reduced and that if $s=n-1$ or $s=n$, then $H^{\prime}$ is upper-Hessenberg. Moreover $s$ needs not be chosen so that $x_{s}$ is the largest component of $x$ (in absolute value). Indeed, since the size of the matrix to be reduced to upper-Hessenberg form increases when $s$ approaches 1 , it is more advantageous to choose the largest $s$ for which the ratios $x_{i} / x_{s}$ are moderate. We wish therefore to define a threshold $t$ for the size of these ratios on the basis of which $s$ will be determined. 
Let $\tilde{H}$ be the computed form of the matrix in (9). In [16], chap. 9, Wilkinson established that if $\|x\|_{\infty} \leq 1$, then the eigenvalues of $\tilde{H}$ are the exact eigenvalues of a matrix $\tilde{H}^{\prime}$ satisfying

$$
\left\|H-\tilde{H}^{\prime}\right\|_{\infty} \leq\|r\|_{\infty}+(n-1) \epsilon
$$

where $r$ is the residual $\lambda x-H x$ and $\epsilon$ is the machine epsilon. With no assumptions on the infinity norm of $x$, this inequality becomes

$$
\left\|H-\tilde{H}^{\prime}\right\|_{\infty} \leq\|r\|_{\infty}+(n-1)\|x\|_{\infty} \epsilon
$$

Since in our algorithm, our computed eigenpairs have residuals on the order of $n\|H\|_{\infty} \epsilon$, we propose $\|H\|_{\infty}$ as a value for $t$.

In addition to destroying the struciure of the matrix, this last method of deflation suffers from the fact that in the case when the eigenvalue to be deflated is non-real, the resulting matrix $H^{\prime}$ is complex and therefore will considerably increase the cost of finding subsequent eigenpairs if a Newton process is restarted from a real initial guess.

\subsection{Deflation with help from the left eigenvector}

The method we introduce now is different in spirit from the ones in the previous section, in that no attempt is made at modifying the matrix.

Assume that $(x, \lambda)$ is an exact eigenpair of $H$ and that $\lambda$ is simple:

$$
H x=\lambda x .
$$

No assumption is made about the remaining eigenvalues of $H$.

Let $(x, X)$ be such that

$$
(x, X)^{-1} H(x, X)=\left(\begin{array}{cc}
\lambda & 0 \\
0 & J
\end{array}\right)
$$

where the right hand side is the Jordan canonical form of $\mathrm{H}$. Now set

$$
(x, X)^{-1}=\left(\begin{array}{c}
y^{H} \\
Y
\end{array}\right)
$$


Then it is clear that $y^{H}$ is a left eigenvector of $H$ corresponding to $\lambda$ and furthermore that

$$
y^{H} X=0 \text {. }
$$

This property can be used to modify Newton's method to avoid converging to the eigenpair $(x, \lambda)$ a second time. Indeed, given $\lambda$ we can compute the left eigenvector $y^{H}$ corresponding to it and use it to confine the current eigenvector to the space $X$. Therefore we can expect to converge to an eigenvector linearly independent of $x$ and hence corresponding to a different eigenpair (since $(x, \lambda)$ was assumed to be simple). When $(x, \lambda)$ has been obtained once, our algorithm for avoiding it then consists of the following major steps:

Compute the left eigenvector $y^{H}$ corresponding to $\lambda$, with $\|y\|_{2}=1$;

Given the current eigenpair $(z, \mu)$ compute the Newton correction from algorithm 2.1;

Let $z^{\prime}$ be the approximate eigenvector obtained after adding the Newton correction to $z$, choose the next eigenvector $z^{\prime \prime}$ as:

$$
z^{\prime \prime}=\left(I-y y^{H}\right) z^{\prime}
$$

In the last step we are just projecting $z^{\prime}$ onto the space $X$. The need might arise to do more than one projection if convergence to more than one known eigenvalue is to be avoided.

It is obvious why we need the known eigenpair to be sim.ple for the algorithm just outlined to work. If $\lambda$ is multiple then left eigenvectors are no longer necessarily orthogonal to $X$. In fact, the algorithm will be adversely affected if the eigenvalue $\lambda$ is ill-conditioned, i.e., if $y^{H} x$ is very small. Indeed, in this case, if the current eigenvector $z=\alpha x+X v$, where $v$ is a vector of length $n-1$, then,

$$
\left(I-y y^{H}\right) z=z-\left(y^{H} z\right) y=\alpha x+X v-\left(\alpha y^{H} x\right) y \approx z
$$

showing that $z$ is hardly modified by the projection and therefore suggesting that the algorithm will not necessarily prevent a second convergence to $(x, \lambda)$. 
The algorithm generalizes to the case when $\lambda$ is multiple in the following way: let $V$ be a right invariant subspace corresponding to $\lambda$. Let $\left(V, V_{c}\right)$ be such that

$$
\left(V, V_{c}\right)^{-1} H\left(V, V_{c}\right)=\left(\begin{array}{cc}
J_{\lambda} & 0 \\
0 & J
\end{array}\right)
$$

where the right hand side is again the Jordan canonical form of $H, J_{\lambda}$ is the Jordan block corresponding to $\lambda$; since $H$ is assumed to be unreduced, there can be only one such block. If we set

$$
\left(V, V_{c}\right)^{-1}=\left(\begin{array}{c}
U^{H} \\
W
\end{array}\right)
$$

then clearly $U^{H}$ is a left invariant subspace corresponding to $\lambda$ and furthermore

$$
U^{H} V_{c}=0
$$

This last property will allow $U^{H}$ to be used in much the same way the left eigenvector was used earlier. However, the practical usefulness of this method is restricted to the case when the eigenvalue $\lambda$ is simple: indeed, the problem of determining the invariant subspace associated with a multiple eigenvalue $\lambda$ is an extremely difficult one and can be prohibitively expensive.

This method in its simplest form (using the left eigenvector) adds $O\left(n^{2}\right)$ work to the cost of finding one eigenpair distinct from $(x, \lambda)$ : this is the cost of computing the left eigenvector corresponding to $\lambda$; the cost of a single projection is $O(n)$.

\subsection{Deflation with orthogonal transformations}

We present now a very stable method for obtaining an upper-Hessenberg matrix $H^{\prime}$ with the property that it has all the eigenvalues of $H$ except for $\lambda[16]$. We assume for now that the eigenpair $(x, \lambda)$ is exact.

The strategy consists of $n-1$ major steps, at each of which a new zero is introduced in tine last column of $H-\lambda I$ starting from the bottom. The configuration at the beginning of the $r^{\text {th }}$ 
step looks like

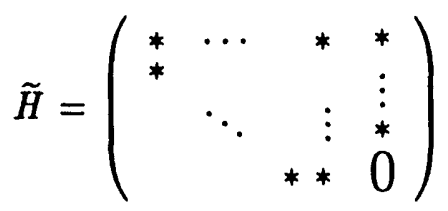

with zeros in the last $r-1$ components of the last column. The $r^{\text {th }}$ step then consists in a post-multiplication by $G_{r}$, where $G_{r}$ is the (possibly complex) rotation in the plane $(n-r, n)$ designed to annihilate the $(n-r, n)$ element:

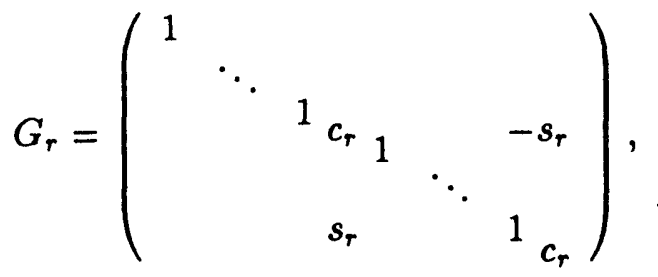

where $\left\|c_{r}\right\|^{2}+\left\|s_{r}\right\|^{2}=1$. This post-multiplication will affect columns $n-r$ and $n$ only and therefore will not disturb zeros previously introduced in the last column. At the $(n-1)$ step, $G_{n-1}$ which is constructed to zero the $(2, n)$ element, will also zero the $(1, n)$ element. Indeed, assume that after the $(2, n)$ element has been zeroed we have some value $\alpha$ in the $(1, n)$ position; then we have

$$
(H-\lambda I) G_{1} \ldots G_{n-1}=\left(\begin{array}{rrrr}
* & \ldots & * & \alpha \\
* & & & \alpha \\
& \ddots & \vdots & 0 \\
& & * * &
\end{array}\right) .
$$

Now if we develop the determinant of this matrix by the last column we get

$$
\operatorname{det}\left[(H-\lambda I) G_{1} \ldots G_{n-1}\right]=\alpha b_{1} \ldots b_{n-1}
$$

where $b_{r}$ is the $r^{\text {th }}$ subdiagonal element of $(H-\lambda I) G_{1} \ldots G_{n-1}$.

$$
\operatorname{det}\left[(H-\lambda I) G_{1} \ldots G_{n-1}\right]=\operatorname{det}(H-\lambda I)=0
$$

since $\left(\operatorname{det}\left(G_{r}\right)=1\right)$ for $r=1, \ldots, n-1$. But $b_{r} \neq 0$ for all $r$, since we have assumed that $H$ had no zeros on the subdiagonal and since post-multiplication by a $G_{r}$ cannot but increase the modulus of a subdiagonal element in $H-\lambda I$. Thus we must have $\alpha=0$ and therefore at the end of the $n-1$ steps just described the last column is zero. Let us set $\mathcal{G}=G_{1} \ldots G_{n-1}$ to simplify the nootatiơn. Theñ,

$$
\mathcal{G}^{-1}=\mathcal{G}^{H}=G_{n-1}^{H} \ldots G_{1}^{H},
$$


and it is straightforward to verify that the zeros of the last column of $(H-\lambda I) \mathcal{G}$ will be preserved when it is pre-multiplied by $\mathcal{G}^{-1}$, because the successive pre-multiplications by $G_{r}^{T}, r=$ $1, \ldots, n-1$, will preserve those zeros. Therefore the last collumn of

$$
\tilde{H}=\mathcal{G}^{H}(H-\lambda I) \mathcal{G}+\lambda I
$$

is equal to $\lambda e_{n}$. The eigenvector of $\widetilde{H}$ corresponding to $\lambda$ is $e_{n}$. Note that $\widetilde{H}$ is not upperHessenberg in this case though. Indeed, non zero elements will be introduced in the last row of $\widetilde{H} ;$ in fact ,

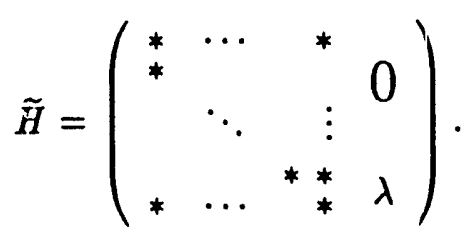

This is not disturbing since if $H^{\prime}$ is the leading principal submatrix of $\tilde{H}$ of order $n-1$, then $H^{\prime}$ is upper-Hessenberg and $\sigma\left(H^{\prime}\right)=\sigma(H)-\{\lambda\}$.

If $\lambda$ is a multiple eigenvalue of $H$ of (algebraic) multiplicity $m$ then $\lambda$ is an eigenvalue of $H^{\prime}$ of multiplicity $m-1$.

So far we have assumed that the eigenpair $(x, \lambda)$ is exact. In practice, $(x, \lambda)$ will only be approximate, in the sense that $H x-\lambda x=r$, is of the order of the machine $\epsilon$. In this case roundoff errors will in general prevent $G_{n-1}$ from annihilating the $(1, n)$ entry. In fact, the accuracy of the computed eigenvalue $\lambda$ will come into play. If $\lambda$ corresponds to an ill-conditioned eigenvalue of $H$, then it is possible that $\lambda$ be a rather poor approximation of the exact eigenvalue. As a consequence the $(1, n)$ entry might not be negligible at all and examples do exist where this is indeed the case [16]. A way around this difficulty is to construct the plane rotations $G_{1}, \ldots, G_{n-1}$ in a way to reduce the vector $x$ to $e_{n}$ and then apply the corresponding similarity transformation to $H$. Inequality (10) from section 3.1 holds when this is done (with obvious modification in the definition of $H^{\prime}$ ). However, this will in general result in introducing non-zero entries below the subdiagonal of $H$ and therefore $H$ needs to be reduced to upper-Hessenberg form again. We reier the reader to [2] for an example of such an algorithin; the geñeralization of that algorithm to the non-real case is straightforward. 
In addition to the difficulty just mentioned, the deflation with plane rotations, suffers from the fact that the deflated matrix will be complex if the eigenvalue to be deflated is complex. Indeed, it is unfortunately not true in general that when an eigenvalue and its complex conjugate are deflated by this method, the resulting matrix is real.

Example 3.1 When the two complex eigenvalues of the $4 \times 4$ upper-Hessenbery matrix,

$$
\left(\begin{array}{cccc}
0.2190 & -0.0756 & 0.6787 & -0.6391 \\
-0.9615 & 0.9032 & -0.4571 & 0.8804 \\
0 & -0.3822 & 0.4526 & -0.0641 \\
0 & 0 & -0.1069 & -0.0252
\end{array}\right)
$$

are deflated using plane rotations as just described we obtain

$$
\left(\begin{array}{cccc}
1.1593-0.0000 i & 0.0000+1.0129 i & 0.0000+0.0000 i & 0.0000+0.0000 i \\
0.0000-0.3053 i & 0.1740-0.0600 i & 0.0000+0.0000 i & 0.0000+0.0000 i \\
-0.1534-0.3540 i & 0.5717+0.0112 i & 0.1082-0.4681 i & 0.0000+0.0000 i \\
-0.4640+0.1988 i & 0.2187-0.3465 i & 0.3964+0.0611 i & 0.1082+0.4681 i
\end{array}\right) .
$$

After the deflation, we will be working with the upper $2 \times 2$ block of $H^{\prime}$ which clearly is complex.

\subsection{Remarks on identifying duplicate eigenvalues}

We have already remarked that the computed eigenpairs from wii algorithm are the exact eigenpairs of a nearby matrix. Under those conditions ([10]),

$$
\left|\lambda-\lambda_{e}\right| \leq\|E\| /\left|s\left(\lambda_{e}\right)\right|
$$

where $E$ is the error in the matrix, and $\left|s\left(\lambda_{e}\right)\right|$ is the ondition number of the exact eigenvalue $\lambda_{e}$ of the original matrix $H$. When $\lambda_{e}$ is ill-conditioned we can expect unpredictably large errors (compared to the tolerated size of the residual) in the computed approximations to $\lambda_{e}$ and therefore in the duplicates if any and identifying these duplicates becomes a rather daunting task: in particular, they will not be detected if their difference is compared to tol introduced earlier. Conversely, it can happen that under certain conditions the tolerated size of the residual be much larger than the distance between certain exact eigenvalues and therefore between certain computed eigenvalues. In this case, it could happen that distinct computed eigeñvalues will be declared as duplicates if their difference is compared to tol. 
Example 3.2 We illustrate this last case with the following matrix

$$
H=\left(\begin{array}{ccc}
2 \cdot 10^{-7} & 0 & 0 \\
2 & 10^{-7} & 0 \\
0 & 2 & 10^{10}
\end{array}\right) \text {. }
$$

The tolerated size of the residuals for this matrix as chosen in our algorithm is tol $\approx\|H\| \epsilon>$ $10^{-6}$. Therefore if we decide to declare as duplicates those eigenvalues whose difference is less than tol then the other two distinct eigenvalues of $H$ will be declared as duplicates.

The problems we have just raised do not have easy solutions [8], and indeed more research is needed here.

\section{Convergence}

In section 2, we mentioned that computing an eigenpair of $H$ reduces to computing a zero of

$$
F_{s}(x, \lambda)=\left(\begin{array}{c}
H x-\lambda x \\
e_{s}^{T} x-1
\end{array}\right) \text {. }
$$

The Jacobian of $F_{s}$ at $(x, \lambda)$ is

$$
F_{s}^{\prime}(x, \lambda)=\left(\begin{array}{cc}
D_{x}(H x-\lambda x) & D_{\lambda}(H x-\lambda x) \\
D_{x}\left(e_{s}^{T}-1\right) & D_{\lambda}\left(e_{s}^{T}-1\right)
\end{array}\right)=\left(\begin{array}{cc}
H-\lambda I & -x \\
e_{s}^{T} & 0
\end{array}\right),
$$

where $D_{x}(F)$ denotes the derivative with respect to $x$ of the function $F$. In this section, we give sufficient conditions for the convergence of our procedure. The result is a version of the Kantorovich theorem as it applies to our case.

Theorem 4.1 (Wilkinson) Assume $(x, \lambda)$ is an exact zero of $F_{s}$. Then

$$
\left(\begin{array}{cc}
H-\lambda I & -x \\
e_{s}^{T} & 0
\end{array}\right)
$$

is singular if and only if $\lambda$ is multiple.

Proof. Let us assume first that $\lambda$ is a multiple eigenvalue of $H$ and that $x$ is the corresponding (exact) cigenvector, then there exists a nonzero vector $y$ such that

$$
y^{H}(H-\lambda I)=0 \text { and } y^{H} x=0
$$


$y$ is simply a left eigenvector of $H$ corresponding to $\lambda$. Thus, $\left(y^{H} 0\right)$ is a nonzero vector and

$$
\left(y^{H} 0\right)\left(\begin{array}{cc}
H-\lambda I & -x \\
e_{s}^{T} & 0
\end{array}\right)=0 .
$$

This proves that the Jacobian is singular.

Now conversely, if $\left(\begin{array}{cc}H-\lambda I & -x \\ e_{s}^{T} & 0\end{array}\right)$ is singular then there exists a nonzero vector $\left(\begin{array}{c}v \\ \mu\end{array}\right)$ such that

$$
\left(\begin{array}{cc}
H-\lambda I & -x \\
e_{s}^{T} & 0
\end{array}\right)\left(\begin{array}{c}
v \\
\mu
\end{array}\right)=0
$$

But then $v_{s}=0$ and $(H-\lambda I) v=\mu x$. If $\mu=0$, then $v$ is nonzero since $\left(\begin{array}{c}v \\ \mu\end{array}\right) \neq 0$, and so $v$ is an eigenvector that is linearly independent of $x$, since $x_{s}=1$ and $v_{s}=0$. Hence $\lambda$ is a multiple eigenvalue in this case. If $\mu \neq 0$, then $v$ is also nonzero; if it were then we would have

$$
\mu x=(H-\lambda I) v=(H-\lambda I) 0=0
$$

and hence $x=0$, contradicting our assumption on $x$. But $(H-\lambda I)^{2} v=\mu(H-\lambda I) x=0$, and thus $v$ is a nonzero vector that has grade 2. Therefore $\lambda$ is multiple in this case as well.

Remark: Since we are assuming that $H$ is upper-Hessenberg and unreduced, an eigenvalue can only be non-derogatory, i.e., the associated eigenspace has dimension one.

The previous result applies to the Jacobian at a zero of $F_{s}$. We wish to know more about the Jacobian at those approximations arising during Newton's iteration and before convergence is declared.

Theorem 4.2 Assume $(x, \lambda)$ is not an exact zero of $F_{s}$. Then $\left(\begin{array}{cc}H-\lambda I & -x \\ e_{s}^{T} & 0\end{array}\right)$ is singular if and only if at least one of the following is true:

1) $\lambda$ is an eigenvalue of $H$ and has an eigenvector whose $s^{\text {th }}$ component is zero.

2) $x$ belongs to the space generated by $\left(c_{1}, \ldots, c_{s-1}, c_{s+1}, \ldots, c_{n}\right)$, where $c_{i}$ is the $i^{\text {th }}$ column of $H-\lambda I$ ( $\lambda$ may or may not be an eigenvalue). 
Proof. Assume first that 1 ) is true and let $y$ be an eigenvector, $y_{s}=0$. Then $\left(\begin{array}{l}y \\ 0\end{array}\right)$ is clearly in the null space of the Jacobian. If 2) holds, and $x=(H-\lambda I) y$, with $y_{s}=0$, then $\left(\begin{array}{l}y \\ 1\end{array}\right)$ is in the null space of the Jacobian.

Conversely, if the Jacobian is singular then there exists a vector $\left(\begin{array}{c}y \\ \mu\end{array}\right)$ such that,

$$
\left(\begin{array}{cc}
H-\lambda I & -x \\
e_{s}^{T} & 0
\end{array}\right)\left(\begin{array}{c}
y \\
\mu
\end{array}\right)=0 .
$$

This implies that

$$
\left\{\begin{array}{c}
(H-\lambda I) y=\mu x \\
e_{s}^{T} y=0
\end{array}\right.
$$

and clearly $y_{s}=0$. We now consider two cases according to whether $\mu$ is zero or not. If $\mu$ is zero, then $\lambda$ is an eigenvalue with corresponding eigenvector $y$, therefore we are in case 1$)$. If $\mu$ is not zero then $\mu x$ and hence $x$ is in the range of $H-\lambda I$ and therefore we are in case 2) since $y_{s}=0$. Note that $\lambda$ may or may not be an eigenvalue in this last case.

Remarks: The theorem tells us that more often than not, a singular Jacobian is an indication that we already have an eigenvalue, and this has been our experience indeed. The singularity of the Jacobian is also an indication of an ill-conditioned eigensystem. In fact, if we accept that the current eigenvector was moving in the "right" direction then if it satisfies condition 2 of the theorem, we can say that the eigenvalue is acting like a multiple one since the eigenvector is also in the range of $(H-\lambda I)$. In practice we have not encountered a situation where condition 1 applied: this is understandable again if we accept that the eigenvector is moving in the right direction since then the eigenvalue would be acting like an eigenvalue of geometric multiplicity more than one, which is impossible since the matrix is unreduced (recall that $x_{s}=1$ ).

The second derivative of $F_{s}$ is a constant bilinear operator with norm equal to 2 . In fact,

$$
F_{s}^{\prime \prime}(x, \lambda)=\left(\begin{array}{cc|cc}
0 & -\mathrm{I} & -\mathrm{I} & 0 \\
0 & 0 & 0 & 0
\end{array}\right) .
$$

Suppose now that our procedure is started with initial guess $\left(x_{0}, \dot{\lambda}_{0}\right)$. We now give sufficient conditions for the convergence of our procedure with the given initial guess. This is provided by 
the classical Kantorovich theorem [5]. Let us first introduce

$$
K=\left\|F_{s}^{\prime-1}\left(x_{0}, \lambda_{0}\right)\right\|
$$

and

$$
c_{1}=\left\|\left(x_{1}, \lambda_{1}\right)-\left(x_{0}, \lambda_{0}\right)\right\|
$$

where $\left(x_{1}, \lambda_{1}\right)$ is the first iterate, i.e.,

$$
\left(x_{1}, \lambda_{1}\right)^{T}=\left(x_{0}, \lambda_{0}\right)^{T}-F_{s}^{\prime-1}\left(x_{0}, \lambda_{0}\right) F_{s}\left(x_{0}, \lambda_{0}\right) .
$$

We call $\left(x_{0}, \lambda_{0}\right), \ldots,\left(x_{k}, \lambda_{k}\right)$ the sequence of iterates produced by the algorithm.

Theorem 4.3 If $\beta_{0}=K c_{1}<1 / 4$, then the sequence $\left(x_{k}, \lambda_{k}\right)$ converges quadratically starting from $\left(x_{0}, \lambda_{0}\right)$.

The process can be regarded as starting from any of the iterates $\left(x_{i}, \lambda_{i}\right)$ and in fact will often converge even when the conditions of the theorem are not satisfied at $\left(x_{0}, \lambda_{0}\right)$. These conditions will then be met for some $\left(x_{k}, \lambda_{k}\right)$ at which stage convergence becomes quadratic.

\section{Defective Case}

Ours being a non-stationary iteration (ihe iterating map is not fixed), it is not easy to analyze the behaviour of the successive approximations. We try however to address this problem in this section with a particular emphasis on the case when either the matrix $H$ or the modified matrix $H_{0}$ is defective, i.e., when either one of these matrices dces not have a complete set of eigenvectors. As we remarked earlier, an eigenvalue of $H$ (with no zeros on the subdiagonal) can only have geometric multiplicity one, and therefore $H$ is defective whenever it has a multiple eigenvalue. An eigenvalue of $H_{0}$ on the other hand, can have geometric multiplicity one or two. In what follows $n$ is the order of $H$. 
The connection between Newton's method and inverse iteration is well known [13]. We present a proof which motivates the subsequent analysis: we let $J$ be the Jacobian of the map $F_{s}$ at $(x, \lambda)$ defined in section 2 ,

$$
J=\left(\begin{array}{cc}
H-\lambda I & -x \\
e_{s}^{T} & 0
\end{array}\right),
$$

and we assume that $x_{s}=1$. The order of the Jacobian is $n+1$. Then,

$$
J=J_{0}+e_{n+1} v^{T}
$$

with

$$
J_{0}=\left(\begin{array}{cc}
H-\lambda I & -x \\
0 & 1
\end{array}\right)
$$

and,

$$
v=e_{s}-e_{n+1}=\left(\begin{array}{c}
0 \\
\vdots \\
0 \\
1 \\
0 \\
\vdots \\
-1
\end{array}\right) .
$$

Assume $J_{0}$ it is not singular; this is true if and only if $\lambda$ is not an eigenvalue. Assume also that $J$ is not singular. The correction to $(x, \lambda)$ is computed in the following manner:

$$
\left(\begin{array}{c}
y \\
\mu
\end{array}\right)=J^{-1}\left(\begin{array}{l}
r \\
0
\end{array}\right),
$$

where $r=\lambda x-H x$. Using the Sherman-Morrison-Woodbury formula [10] we can write $J^{-1}$ as,

$$
J^{-1}=\left(J_{0}+e_{n+1} v^{T}\right)^{-1}=J_{0}^{-1}-\frac{1}{1+v^{T} J_{0}^{-1} e_{n+1}} J_{0}^{-1} e_{n+1} v^{T} J_{0}^{-1}
$$

Therefore, letting $b=\left(\begin{array}{l}r \\ 0\end{array}\right)$, we have,

$$
\left(\begin{array}{c}
y \\
\mu
\end{array}\right)=J^{-1} b=J_{0}^{-1} b-\frac{v^{T} J_{0}^{-1} b}{1+v^{T} J_{0}^{-1} e_{n+1}} J_{0}^{-1} e_{n+1} .
$$

It can be easily checked chat

$$
J_{0}^{-1} b=\left(\begin{array}{c}
-x \\
0
\end{array}\right)
$$

and that

$$
J_{0}^{-1} e_{n+1}=\left(\begin{array}{c}
\bar{x} \\
1
\end{array}\right)
$$


where $(H-\lambda I) \tilde{x}=x$. Now if we let $\left(x_{1}, \lambda_{1}\right)$ be the next eigenpair we have from (11) and the subsequent equalities,

$$
\left(\begin{array}{l}
x_{1} \\
\lambda_{1}
\end{array}\right)=\left(\begin{array}{l}
x \\
\lambda
\end{array}\right)+\left(\begin{array}{c}
y \\
\mu
\end{array}\right)=\left(\begin{array}{c}
x \\
\lambda
\end{array}\right)+\left(\begin{array}{c}
-x \\
0
\end{array}\right)-\frac{v^{T} J_{0}^{-1} b}{1+v^{T} J_{0}^{-1} e_{n+1}}\left(\begin{array}{c}
\tilde{x} \\
1
\end{array}\right) .
$$

Furthermore,

$$
\frac{v^{T} J_{0}^{-1} b}{1+v^{T} J_{0}^{-1} e_{n+1}}=\frac{-1}{\tilde{x}_{s}}
$$

and so finally we see that our scheme reduces to the following: Given $(x, \lambda)$ compute the next iterate $\left(x_{1}, \lambda_{1}\right)$ via

$$
\begin{gathered}
(H-\lambda I) \tilde{x}=x \\
x_{1}=\frac{1}{\tilde{x}_{0}} \tilde{x} \\
\lambda_{1}=\lambda+\frac{1}{\tilde{x}_{0}} .
\end{gathered}
$$

\subsection{Case when $H$ is defective}

When the algorithm is expressed as in (12) we can readily see some of the difficulties that arise when the matrix $H$ is defective or almost defective, which is more likely in general due to roundoff errors. These problems are similar to the kind of problems that face the application of inverse iteration. More precisely, assume that $H$ is almost defective with $x_{1}, \ldots, x_{n}$ as a complete set of eigenvectors. Let $\lambda_{1}, \ldots, \lambda_{\ell}$ be a cluster of eigenvalues of $H$ and suppose that the initial approximate eigenvalue $\lambda$ corresponds to one of these. The eigenvectors $x_{1}, \ldots, x_{\ell}$ corresponding to these eigenvalues are then almost linearly dependent. In general, the eigenvector corresponding to $\lambda$ can be expected to converge to the space generated by $x_{1}, \ldots, x_{\ell}$. As the eigenvalue $\lambda$ approaches the cluster however, continued rorrections to the eigenvector cannot be expected to refine it. We refer the reader to the particrlarly lucid account in [13] for a justification of these claims. Solving as in inverse iteration (see INVIT [14]) is a possible way around this problem. Computing the residual with extended precision arithmetic is also an obvious approach, and has been successful in practice.

When approaching a singular solution, Newton's method loses its quadratic convergence rate. We have proved earlier (Thm. 4.1) that the Jacobian is singular at multiple eigenpairs and 


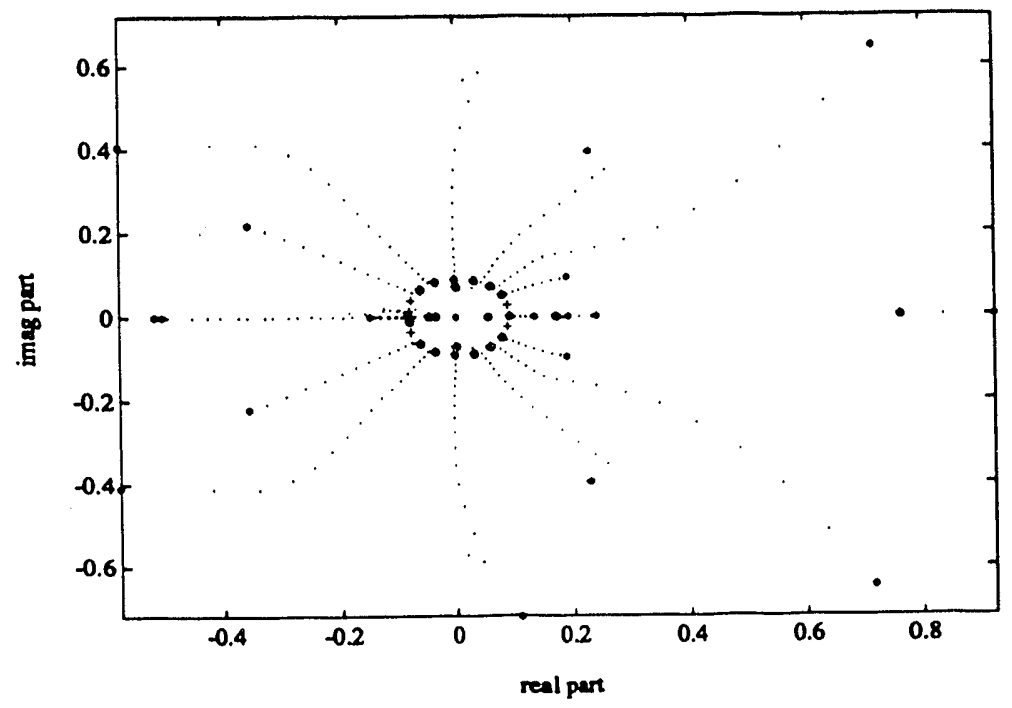

Figure 1: The behavior of the algorithm for an almost defective $25 \times 25$ matrix. The crosses are the eigenvalues of the original matrix; the stars are the initial guesses; the circles are the eigenvalues computed by our algorithm; the dots are the iterates arising in Newton's iterations.

therefore we can expect slower convergence when these are the target (see Fig. 1).

Recall the rate of change of $\lambda$ that we derived in section 2 :

$$
\left|\lambda^{\prime}(0)\right|=\frac{|\alpha|\left|y_{k+1}\right|\left|x_{k}\right|}{\left|y^{H} x\right|}
$$

We wish to caution from hastily drawing conclusions about the sensitivity of the eigenvalues of a defective matrix to our dividing process from this expression. Indeed, as an extreme case which will help illustrate our point, the eigenvalues of a defective matrix can remain virtually unchanged after a zero has been introduced in the subdiagonal. An example, is the $2 \times 2$ matrix

$$
\left(\begin{array}{ll}
1 & 0 \\
1 & 1
\end{array}\right)
$$

After the dividing process we have

$$
\left(\begin{array}{ll}
1 & 0 \\
0 & 1
\end{array}\right)
$$

The starting eigenpairs are then $\left(\left(\begin{array}{l}1 \\ 0\end{array}\right), 1\right)$ and $\left(\left(\begin{array}{l}0 \\ 1\end{array}\right), 1\right)$. The second of these is of course the exact eigenpair. Now, even though the first starting eigenvector is orthogonal to the desired one, the equations arising during the first of Newton's iterations can be solved in a way to produce 
the desired eigenvector from the first step: zero pivots need to be replaced by small numbers on the order of the machine unit roundoff (as is done in inverse iteration, INVIT [14]).

Finally, we mention that the deflation process can contribute to the improvement of the condition of the eigenvalues. Indeed, if $\lambda$ and $\lambda^{\prime}$ are pathologically close (and fairly distant from the rest of the eigenvalues), then by deflating $\lambda$, say, $\lambda^{\prime}$ will have a better condition number as an eigenvalue of the resulting matrix. Indeed, $\lambda^{\prime}$ is not part of a cluster anymore.

\subsection{Case when $H_{0}$ is defective}

It can happen in this case (e.g., if $H$ is non-defective) that the initial dividing process would leave us with a number of initial approximations that is smaller than $n$. This will inhibit the parallelism of the algorithm in that less Newton processes can be started simultaneously. Furthermore, whatever eigenpairs we have can be extremely poor approximations to the desired ones. An extreme situation is illustrated by the matrix

$$
H=\left(\begin{array}{llll}
0 & 0 & 0 & 1 \\
1 & 0 & 0 & 0 \\
0 & 1 & 0 & 0 \\
0 & 0 & 1 & 0
\end{array}\right)
$$

No matter where the zero is introduced on the subdiagonal, the resulting matrix has 0 as its only eigenvalue, and we only have two initial approximations to the four distinct eigenpairs of $H$, namely:

$$
\left(\left(\begin{array}{l}
0 \\
1 \\
0 \\
0
\end{array}\right), 0\right), \quad\left(\left(\begin{array}{l}
0 \\
0 \\
0 \\
1
\end{array}\right), 0\right)
$$

if the zero is introduced in the $(3,2)$ position.

Furthermore, the Jacobian is exactly singular at each of these initial approximations. Indeed 
the Jacobian at $\left(\left(\begin{array}{l}0 \\ 1 \\ 0 \\ 0\end{array}\right), 0\right)$ is

$$
\left(\begin{array}{ccccc}
0 & 0 & 0 & 0 & 0 \\
1 & 0 & 0 & 0 & -1 \\
0 & 1 & 0 & 0 & 0 \\
0 & 0 & 1 & 0 & 0 \\
0 & 1 & 0 & 0 & 0
\end{array}\right)
$$

which is clearly of rank three since the first and the last columns are linearly dependent, and the fourth column is zero. Similarly it can be seen that the Jacobian at $\left.\left(\begin{array}{l}0 \\ 0 \\ 0 \\ 1\end{array}\right), 0\right)$ is also singular. A remedy to this situation is to perturb the initial guess zero by a small amount thereby making the Jacobian non singular, and indeed this has been successful in practice. The problem in this case, as in most other similar cases, results from the particular structure of the matrix. In order to obtain further eigenvalues, we need to deflate the matrix each time a new eigenpair is computed which makes the algorithm almost serial.

\subsection{Known failures}

Some matrices of the structure mentioned at the end of section 5.2 (companion-like matrices), provided us with the only cases where the algorithm failed in practice to converge to the desired eigenpairs, i.e., failed to produce eigenpairs with small residuals after a fixed number of iterations. Should these matrices be subjected to orthogonal similarity transformations however and then reduced back to upper-Hessenberg form, the dividing process will provide us with much better initial approximations and indeed, will converge for all initial approximations. We are certainly not advocating this as a viable scheme: we wanted to emphasize the fact that it is the structure of the matrix that caused the poor approximations and the failures, and not some inherent difficulty with the spectrum of these matrices. 


\section{Work Estimates}

We assume in this section that we are given a real dense matrix $A$. Then the first task in our algorithm is to reduce $A$ to an upper-Hessenberg matrix $H$. This requires $\frac{14 n^{3}}{3}$ operations (plus lower order terms) since the orthogonal matrices used in the reduction are to be accumulated $[10]$.

The dividing process is now applied to $H$. Assume $n=2^{m} r$, for some $m \geq 1$ where $r$ is not necessarily relatively prime to 2 . If we repeat the dividing process $m$ times, we end up with $2^{m}$ matrices of size $r$, each of which is upper-Hessenberg. A submatrix of size $\frac{n}{2^{\prime}}$ obtained in the dividing process will be referred to as a matrix at the level $i$. The matrix $H$ itself is at the level 0 , and the $r$ matrices referred to above are at the level $m$. Thus, we have $m+1$ levels in total. Let $p^{\prime}=2^{m}$ be the number of submatrices at the lowest level, and $p$ the number of processors; we will assume that $p=2^{q}, q \leq m$. The cost of finding the eigenpairs of a (Hessenberg) matrix at the lowest level (by the QR algorithm) is roughly $18\left(\frac{n}{p^{\prime}}\right)^{3}:$ this figure is very approximate and assumes among other things that two QR steps are needed before a real or two complex conjugate eigenvalues are identified and that the matrix has an equal number of real and complex eigenvalues [10]. Let $s_{\ell}=\frac{n}{2^{\ell}}$ be the size of one matrix at the level $\ell$. Let $k_{\ell}$ be the average number of iterations needed to get one eigenpair of a matrix at the level $\ell$. $k_{\ell}$ depends on the matrix and on its size of course. We will make the following simplifying assumption however: $k_{\ell}=k, \ell=0, \cdots, m$, i.e., we assume that the average number of steps required for convergence is the same at all levels. Our experience with the algorithm suggests that this is a realistic assumption as long as the size of the submatrices remains moderate. If the number of levels is increased to the point where we are left with small submatrices, then $k_{\ell}$ becomes significantly larger as $\ell$ increases.

The cost of computing one correction at the level $\ell$ is roughly $6 s_{\ell}^{2}$ : indeed, one Newton iteration involves the solution of a linear system that is upper-Hessenberg, but for possible non- 
zeros in the last row; the order of this linear system is $s_{\ell}+1$. Therefore 2 multipliers at most need be computed per column and, when updating the matrix, each of these will be used in $2(s \ell+1-i)$ multiplications and additions, where $i$ is the index of the column. The factorization of the Jacobian requires then $2 s_{\ell}^{2}$ operations in addition to $O\left(s_{\ell}\right)$ operations (including divisions and comparisons). The forward solve is $O\left(s_{\ell}\right)$ work and is negligible. The backsolve requires $s_{\ell}^{2}$ operations and computing the residual requires another $s_{\ell}^{2}$ operations. Therefore for a real current approximation one correction comes at the cost of $4 s_{\ell}^{2}$. For a complex current eigenpair, this becomes $16 s_{\ell}^{2}$ since the dominant operations are a roughly equal number of multiplications and additions. Since, as we indicated in section 2, only one of a conjugate pair of complex eigenpairs need be corrected, we can assume that $8 n^{2}$ operations are required for correcting a complex eigenpair. Assuming again that the matrix has an equal number of real and complex eigenvalues, our estimate for the amount of work required for computing one correction at the level $\ell$ becomes $6 s_{\ell}^{2}$ operations.

We shall use $\frac{n}{p}$ initial guesses to start $\frac{n}{p}$ Newton processes on each processor. We now have the following work estimate on one processor, assuming all processors share equally the cost of all the stages of the algorithm

$$
W_{p}=\frac{14 n^{3}}{3 p}+18\left(\frac{p^{\prime}}{p}\right)\left(\frac{n}{p^{\prime}}\right)^{3}+\sum_{\ell=0}^{m-1} 6\left(\frac{n}{p}\right) k s_{\ell}^{2}+2 \frac{n^{3}}{p},
$$

where: $\frac{14 n^{3}}{3 p}$ is the processor's contribution to the reduction of the original matrix to upperHessenberg form; $18\left(\frac{p^{\prime}}{p}\right)\left(\frac{n}{p^{\prime}}\right)^{3}$ is the cost of applying the QR algrithm to $\frac{p^{\prime}}{p}$ matrices of size $\frac{n}{p^{\prime}}$; $\sum_{\ell=0}^{m-1} 6\left(\frac{n}{p}\right) k s_{l}^{2}$ is the cost of solving $k$ linear systems with matrices of size $s_{\ell}$, repeating this for $\frac{n}{p}$ initial guesses and for $\ell=0, \cdots, m-1 ; 2 \frac{n^{3}}{p}$ is the processor's contribution to the computation of the eigenvectors of the original dense matrix $A$ once those of $H$ have been computed. The expression for the work can be rewritten:

$$
W_{p}=\frac{n^{3}}{p}\left(\frac{20}{3}+\frac{18}{p^{\prime 2}}+\frac{24 k}{3}\left(1-\left(\frac{1}{4}\right)^{m}\right)\right) .
$$


But $p^{\prime}=2^{m}$ and therefore $p^{2}=4^{m}$ and hence

$$
W_{p}=\frac{n^{3}}{p}\left(\frac{20}{3}+8 k+(18-8 k) \frac{1}{4^{m}}\right),
$$

where for ease of reference we redefine the various parameters: $n$ is the order of the matrix; $p$ is the number of processors; $k$ is the average number of Newton iterations needed before an eigenpair is accepted; and $m$ is the number of zeros introduced on the subdiagonal.

The cost of getting the eigenvalues and eigenvectors by the QR algorithm is $25 n^{3}$ [10]. A reasonable value for $k$ is 3 , however there are cases when $k$ is 2 or less. There are also cases where $k$ is larger than 3 , mostly with matrices of small order or defective matrices.

It is easy to verify that for $k \leq 2$ and for $m=1$ (one split) the model for the cost of the algorithm predicts sequential speedup over QR. Our model does not predict sequential speedup for problems where $k$ equals or exceeds 3 (see Fig. 2). Here are some sample values of $W_{p}$, assuming that $k=3$ and $p=p^{\prime}=2^{m}$. which means that the original problem is subdivided into a number of problems equal to the number of available processors.

$$
p=128 \rightarrow W_{p}=0.2396 n^{3} ; p=1024 \rightarrow W_{p}=0.0299 n^{3} .
$$

Here we have assumed that the problem is large enough to allow the efficient use of that many processors. Note that the ratio of the work estimate from our model to the work estimate of QR is independent of $n$. This is due to the simplifying assumption on $k$ made at the beginning of this section. That assumption has in effect "hidden" the dependency on $n$ of the coefficient of $n^{3}$ in our model. Figures 2, 3, 4 and 5 show plots of the work estimate for various values of the parameters involved. 


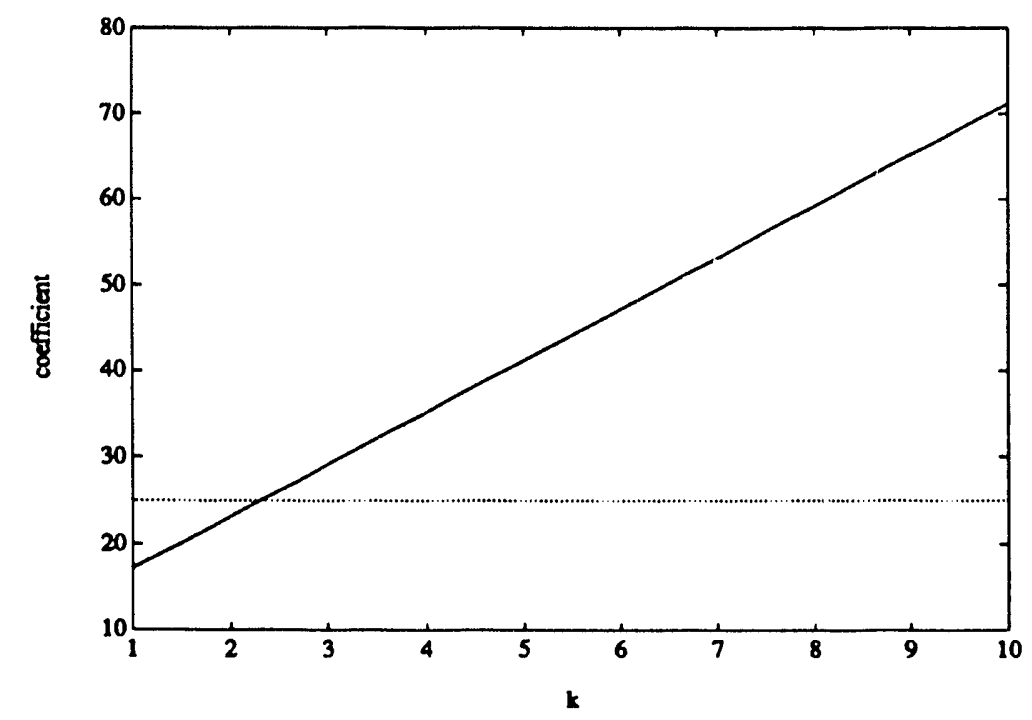

Figure 2: Variation of the Coefficient of $n^{3}$ in work estimate model in terms of the number of steps $k$, with $m=1$, and $p=1$ (see expression for work).

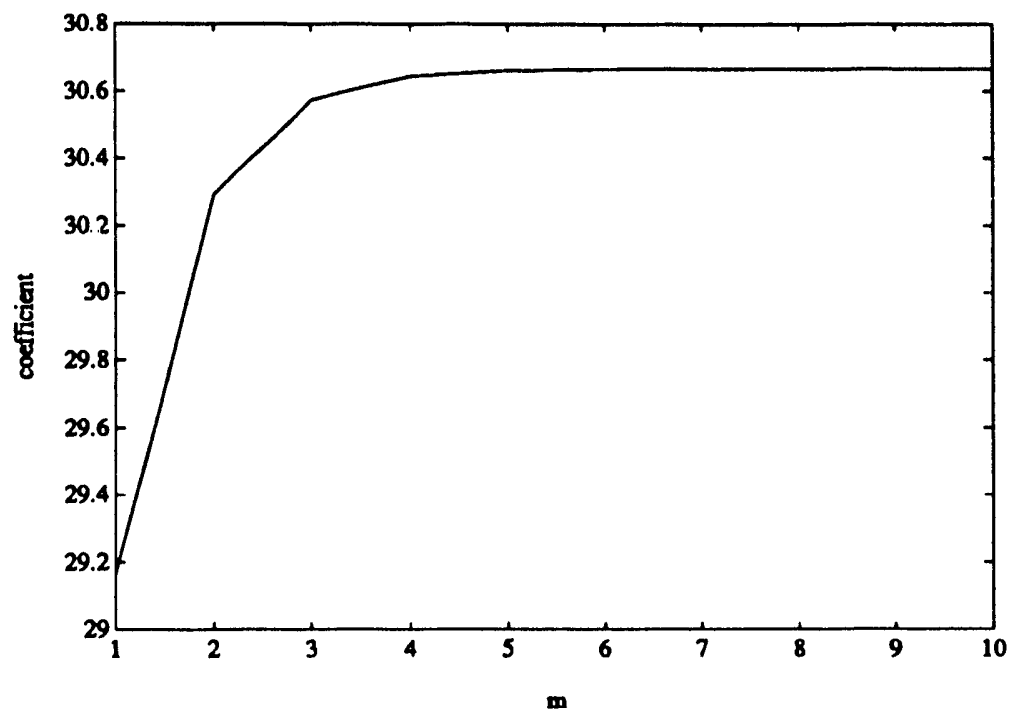

Figure 3: Variation of the Coefficient of $n^{3}$ in work estimate model in terms of the number of splits $m$, with $k=3$ and $p=1$ (see expression for work). 


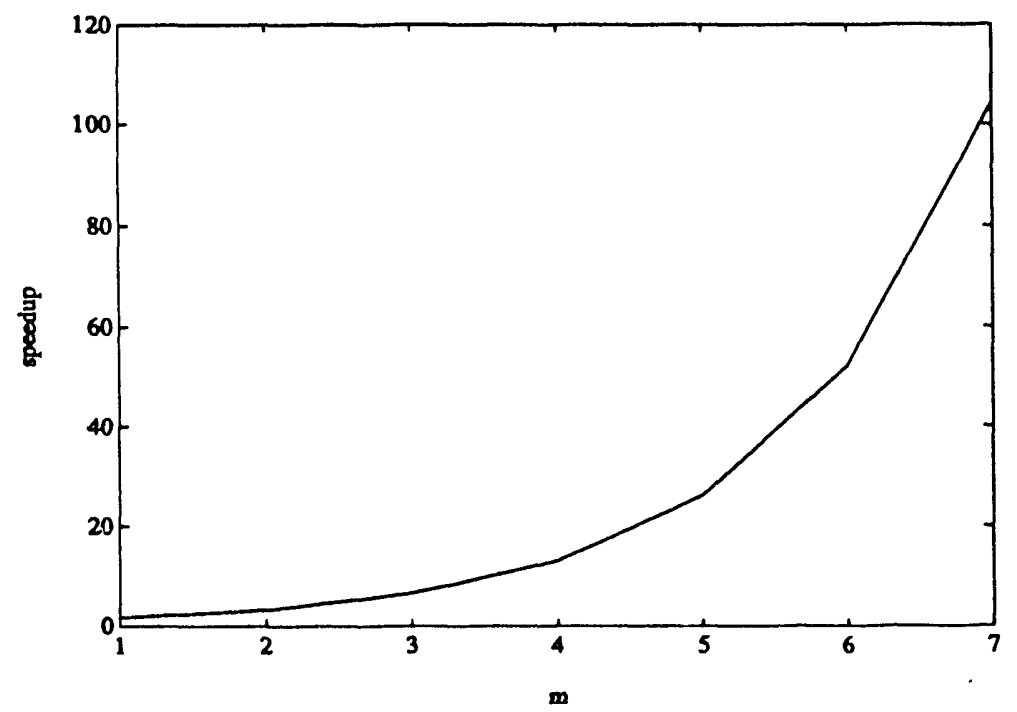

Figure 4: Predicted speedup over QR in terms of number of splits $m$, with $p=2^{m}$ and $k=3$ (see expression for work).

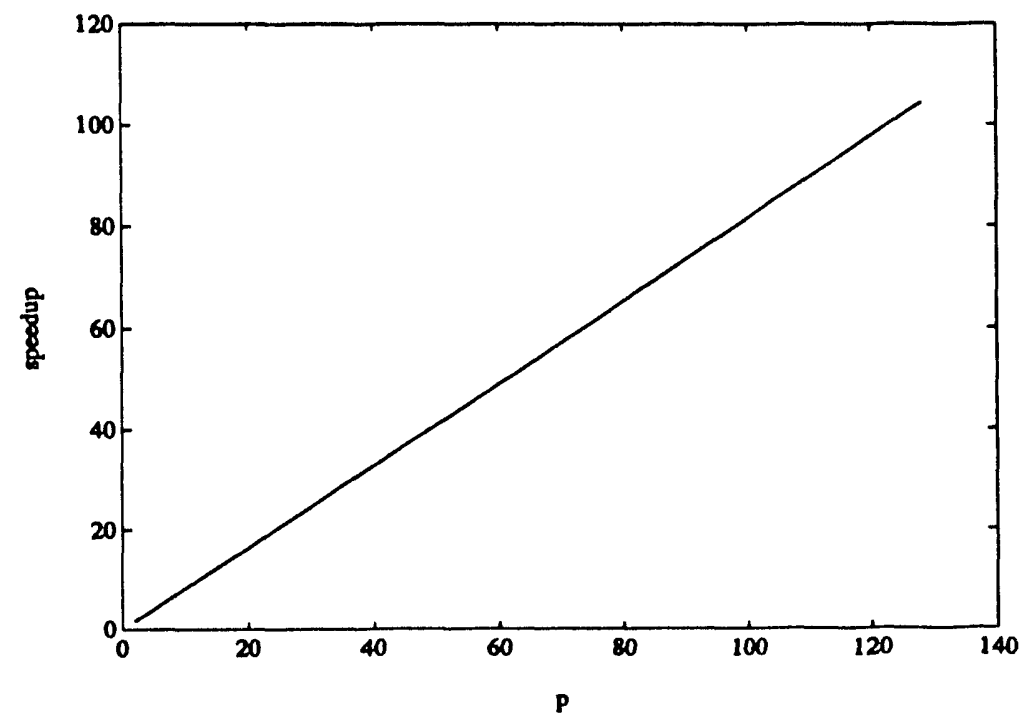

Figure 5: Predicted speedup over QR in terms of the number of processors $p$, with $m=\log _{2} p$ and $k=3$ (see expression for work). 


\section{Parallel Algorithms Details and Performance}

It is fairly straightforward to see from section 2 how to obtain a parallel algorithm. We discuss here certain details. The given, generally dense, matrix is first reduced to upper-Hessenberg form using a blocked algorithm. Next comes the partitioning phase or "divide". This phase amounts to constructing a binary tree with each node representing a partition into two subproblems. It has been our practice to partition the matrix into a number of subproblems (at the lowest level) equal to the number of processors available on the target machine. Each of these problems may be spawned independently without fear of data conflicts; the computation at this level (the lowest) consists of calls to the EISPACK routine HQR2. The tree is then traversed in reverse order with Newton's method applied at each node using the results from the sons as initial approximations. Note here that the computation at a node does not have to wait for both sons to complete in order to start: as a matter of fact, it can start as soon as one son has computed one eigenpair of a subproblem. In order to stress this point, we mention here that this is quite different from the situation in the symmetric case [6], where information from both sons is needed before computations can start at the node. However, in practice we have allowed computations to start at a node only after at least one son has completed; the need to check for duplicate eigenvalues and deflate if necessary has imposed further synchronization.

The algorithm has been implemented on computers with a shared memory and computers with distributed memory architectures.

\subsection{Shared memory implementation}

So far we have used SCHEDULE [7] to implement the algorithm on shared memory computers. SCHEDULE is a package of FORTRAN and C subroutines designed to aid in programming explicitly parallel algorithms for numerical calculations. An important part of this package is the provision of a mechanism for dynamically spawning processes even when such a capability is not present within the parallel language extensions provided for a given machine. 


\subsection{Distributed memory implementation}

The current implementation on distributed memory machines requires that the matrix be stored on each processor. This obviously puts constraints on the size of problems that can be solved. With this implementation however, communication is needed only during the deflation phase. This implementation is best described through the contribution of a particular processor. Suppose that we have 4 processors at our disposal, $p_{0}, \ldots, p_{3}$ and that accordingly the matrix $H$ has been divided into 4 subproblems: $H_{0}, \ldots, H_{3}$, that their common size is $n / 4$ and that they occur in this order on the diagonal of the matrix. We describe now the contribution of $p_{2}$ by steps:

1) Call HQR2 to solve for the eigensystem of the matrix $\mathrm{H}_{2}$.

2) Refine the output from step 1 to get $1 / 2$ the number of (i.e., $n / 4$ ) eigenpairs of the matrix $H_{\frac{1}{2}}:$

$$
H_{\frac{1}{2}}=\left(\begin{array}{c|c}
H_{2} & B \\
\hline 0^{\alpha} & H_{3}
\end{array}\right),
$$

where $H_{\frac{1}{2}}$ is a submatrix of $H$.

3) Refine the output from step 2 to get $1 / 4$ of the number of eigenpairs (i.e., $n / 4$ ) of the matrix $H$.

As can be readily realized no communication between processors is needed except for checking for eigenpairs to which convergence occurred from more than 1 initial approximation: for example, the eigenpairs of $H_{\frac{1}{2}}$ are generated on $p_{2}$ and $p_{3}$, and therefore we need to check for duplicate eigenpairs (on each processor separately which requires no communications, and across both which requires communications).

We are currently developing another implementation where blocks of columns of the matrix are stored on different processors. This storage scheme has been dictated to us by the need to call HQR2 at the lowest level: indeed, the call to HQR2 requires that contiguous columns of the 
matrix reside on the same processor. Therefore storage schemes more advantageous for linear system solving, such as wrap mapping of columns or rows, could not be used. The communication between processors for this implementation is more intensive. Communication is indeed needed when solving the linear systems arising in Newton's iterations as well as for the deflation phase. Also because of the storage scheme, we can expect the processors to become successively idle during the factorization of the Jacobian and the back solve for the correction. However, we have implemented efficiently a scheme where the Jacobian is repartitioned by rows before the back solve takes place: the "reshuffling" of the submatrices takes place between processors that became idle after doing their part of the Gaussian elimination.

\section{Numerical Results and Performance}

In this section we present the results of the implementation of the algorithm on a number of machines. The serial version of the code is available through NETLIB where it's called "nonsymdc".

The same algorithm has been run on the IBM RS/6000/500, the Alliant FX/8, the Intel iPSC/2 and the Intel iPSC/860. We compared our results to those of HQR2 from the EISPACK collection. We have used randomly generated matrices in these tests. In these implementations the linear solver used for the computation of the correction at each Newton step is column oriented. The storage requirement for our algorithm in a serial implementation is $4 n^{2}+O(n)$. The following are the results from the IBM RS/6000/500 implementation. The IBM RS/6000/500 is a single processor computer with a RISC-based Architecture.

\begin{tabular}{c|c|c|c|c} 
Order & HQR2 & D\&C & Ratio HQR2/D\&C & Distinct $\lambda$ \\
\hline 100 & 1.04 & 1.12 & .93 & 99 \\
200 & 9.31 & 9.18 & 1.01 & 196 \\
300 & 34.1 & 28.1 & 1.2 & 293 \\
400 & 94.0 & 65.3 & 1.4 & 395 \\
500 & 196 & 136 & 1.4 & 490 \\
1000 & 1741 & 992 & 1.7 & 1000
\end{tabular}


In an implementation on shared memory machine, the storage allocated to the Jacobian (in serial mode) is multiplied by the number of processors used; this is meant to prevent concurrent write to the same memory locations. The following are some results from the Alliant FX/8 implementation. The Alliant $F X / 8$ is a parallel machine with 8 vector processors.

\begin{tabular}{c|c|c|c} 
Order & No. of procs. & Levels & Ratio HQR2/D\&C \\
\hline 100 & 2 & 1 & 1.7 \\
& 4 & 2 & 2.4 \\
& 8 & 3 & 4.0
\end{tabular}

The results on the Alliant were in general disappointing. The storage scheme for the Jacobian that we used on that machine seems to have inhibited the compiler performed vector optimizations. HQR2, running on a single processor was vector-optimized.

Finally we present results from the runs on the hypercubes. The following are some results from the Intel iPSC/ 2 implementation. The largest size used in each case was dictated by the memory capacity of a single node.

\begin{tabular}{c|c|c|c} 
Order & No. of procs. & Levels & Ratio HQR2/D\&C \\
\hline 100 & 2 & 1 & 2.2 \\
& 4 & 2 & 3.7 \\
& 8 & 3 & 6.0 \\
500 & 16 & 4 & 8.2 \\
& 2 & 1 & 2.2 \\
& 4 & 2 & 3.5 \\
& 8 & 3 & 5.2 \\
& 16 & 4 & 9.1 \\
& 2 & 1 & 2.2 \\
& 4 & 2 & 3.2 \\
& 8 & 3 & 6.3 \\
& 16 & 4 & 9.8 \\
& 32 & 5 & 13.2 \\
& 64 & 6 & 21.3
\end{tabular}

The following are some results from the Intel iPSC/860 implementation. 


\begin{tabular}{c|c|c|c} 
Order & No. of procs. & Levels & Ratio HQR2/D\&C \\
\hline 100 & 2 & 1 & 1.9 \\
& 4 & 2 & 3.3 \\
400 & 8 & 3 & 5.1 \\
& 2 & 1 & 2.4 \\
& 4 & 2 & 3.2 \\
600 & 8 & 3 & 6.0 \\
& 16 & 4 & 8.4 \\
& 8 & 3 & 7.5 \\
& 16 & 4 & 13.5 \\
& 32 & 5 & 23 \\
& 64 & 6 & 32
\end{tabular}

We observe here that the speedups realized by our algorithm over the $Q R$ algorithm, did not remain linear for a large number of processors. This is due to the fact that our algorithm is much less efficient on small matrices and we had to work with this kind of matrices when the number of processors became large. For example, with a matrix of order 600 and using 64 processors, matrices of average size 20 had to be solved on each node at level 5 .

\section{The generalized eigenvalue problem}

We show here how the ideas behind our algorithm can be used to solve the generalized eigenvalue problem.

\subsection{Basic algorithm}

Given an upper-Hessenberg matrix $H$ and an upper-triangular matrix $U$ :

$$
H=\left(\begin{array}{c|c}
H_{11} & H_{12} \\
\hline 0^{\alpha} & H_{22}
\end{array}\right), \quad U=\left(\begin{array}{c|c}
U_{11} & U_{12} \\
\hline 0 & U_{22}
\end{array}\right),
$$

we want to solve the generalized eigenvalue problem,

$$
H x=\lambda U x .
$$

Without loss of generality, we can assume $H$ to be unreduced and $U$ to be non-singular since otherwise the problem reduces to a smaller problem. Then our algorithm generalizes easily. 
Indeed, set

$$
H_{0}=H-\alpha e_{k+1} e_{k}^{T}
$$

and consider solutions of (or approximations of these)

$$
H_{0} x=\lambda U x
$$

as initial approximations to the sought eigenpairs. More precisely, solving (15) reduces to solving

$$
H_{11} x=\lambda U_{11} x \text { and } H_{22} x=\lambda U_{22} x .
$$

Let's denote these solutions by $\left(\tilde{x}_{1}, \lambda_{1}\right), \ldots,\left(\tilde{x}_{n}, \lambda_{n}\right)$ (we have $n$ solutions because of our assumption that $U$ is non-singular). These can be used to construct initial approximations $\left(x_{1}, \lambda_{1}\right), \ldots,\left(x_{n}, \lambda_{n}\right)$ to the eigenpairs of the original generalized eigenproblem in much the same way we did it in the case $U=I$. More precisely, we take

$$
\left(x_{i}, \lambda_{i}\right)=\left(\left(\begin{array}{c}
\tilde{x}_{i} \\
0
\end{array}\right), \lambda_{i}\right)
$$

if $\lambda_{i} \in \sigma\left(H_{11}, U_{11}\right)$ and

$$
\left(x_{i}, \lambda_{i}\right)=\left(\left(\begin{array}{c}
0 \\
\tilde{x}_{i}
\end{array}\right), \lambda_{i}\right)
$$

if $\lambda_{i} \in \sigma\left(H_{22}, U_{22}\right)$ (an appropriate number of zeros in each case).

Finally solving the generalized eigenvalue problem (14) above is the same as solving the problem

$$
\left\{\begin{array}{l}
H x-\lambda U x=0 \\
L(x)=1
\end{array}\right.
$$

where $L(x)$ is a scalar equation, which we take to be a normalizing condition: $e_{s}^{T} x=1$. Then for each initial eigenpair $\left(x_{i}, \lambda_{i}\right)$, successive corrections can be computed via

$$
\left(\begin{array}{cc}
H-\lambda_{i} U & -U x_{i} \\
e_{s}^{T} & 0
\end{array}\right)\left(\begin{array}{c}
y \\
\mu
\end{array}\right)=\left(\begin{array}{c}
r_{i} \\
0
\end{array}\right), \lambda_{i} \leftarrow \lambda_{i}+\mu ; x_{i} \leftarrow x_{i}+y
$$

where

$$
r_{i}=\lambda_{i} U x_{i}-H x_{i}
$$


A possible stopping criterion for this scheme is the condition

$$
\frac{\left\|H x_{i}-\lambda_{i} U x_{i}\right\|}{\left\|x_{i}\right\|} \leq f(n)\|H\|\|U\| \epsilon,
$$

where $f(n)$ is a modest function of $n$. It is easy to see that starting from two complex conjugate initial guesses the algorithm will compute complex conjugate corrections, therefore allowing similar savings to those in the case $U=I$.

\section{Deflation in the generalized case}

The method of deflation presented in section 3.1 generalizes to this case in the following manner.

Let $H x=\lambda U x$ (of course, in practice we only have an approximate eigenpair). Then it is true that if $w=U x$ then $w_{n} \neq 0$ : Indeed, if $w_{n}=0$, then it can be shown that $w$ is zero using the fact that $H$ is unreduced. $U$ being non singular this implies that $x$ is zero which is not true.

Let

$$
\left(\begin{array}{c}
-v \\
1
\end{array}\right)=w / w_{n}
$$

and

$$
u=\left(\begin{array}{c}
v \\
1
\end{array}\right)
$$

We know that $\sigma(H, U)$ is the same as $\sigma\left(M^{\prime} H M, M^{\prime} U M\right)$ for any non-singular $M$ and $M^{\prime}$. For our purposes, we take

$$
M=\left[e_{1}, \ldots, e_{n-1}, x\right]
$$

and

$$
M^{\prime}=\left[e_{1}, \ldots, e_{n-1}, u\right] .
$$

Then it is easy to verify that we have

$$
M^{\prime} H M=\left(\begin{array}{c|c}
H^{\prime} & 0 \\
\hline 0^{\alpha} & \gamma
\end{array}\right), M^{\prime} U M=\left(\begin{array}{c|c}
U^{\prime} & 0 \\
\hline 0 & \delta
\end{array}\right),
$$

where $\bar{H}^{\prime}$ is upper-Hessenberg, $\bar{U}^{\prime}$ is upper-triangular and

$$
\frac{\gamma}{\delta}=\lambda
$$


In fact in this particular case we have

$$
\gamma=\lambda
$$

and

$$
\delta=1
$$

Clearly:

$$
\sigma\left(H^{\prime}, U^{\prime}\right)=\sigma(H, U)-\{\lambda\} .
$$

Therefore, having "removed" $\lambda$ from the spectrum we can get further eigenpairs. We note that, just as in the case $U=I, H^{\prime}$ and $U^{\prime}$ are very cheap to obtain once $M^{\prime}$ has been determined. Indeed, $H^{\prime}$ differs from the $n-1 \times n-1$ principal submatrix of $H$ in the last column only, whereas $U^{\prime}$ is the $n-1 \times n-1$ principal submatrix of $U$. The computation of $M^{\prime}$ requires a matrix-vector multiply.

It is also easy to verify that deflating two consecutive complex conjugate eigenpairs results in real $H^{\prime}$ and $U^{\prime}$.

The condition number of $M^{\prime}$ might raise concern. We have

$$
\operatorname{cond}_{\infty}\left(M^{\prime}\right) \approx \max \left(\left|w_{i}\right|\right)^{2}
$$

It is therefore easy to detect an ill-conditioned $M^{\prime}$. We propose to handle this situation in the generalized case in the following manner. Let $D$ be a diagonal matrix with its diagonal elements $d_{i}$ defined by

$$
\left\{\begin{array}{c}
d_{i}=1, \text { if } \frac{w_{i}}{w_{n}} \leq 1 \\
d_{i}=-\frac{w_{n}}{w_{i}}, \text { if } \frac{w_{i}}{w_{n}}>1
\end{array}\right.
$$

then clearly

$$
\sigma\left(D M^{\prime} H M, D M^{\prime} U M\right)=\sigma(H, U)
$$

since $D$ is non-singular. The multiplication by $D$ cancels all the large entries in the last column of $M^{\prime}$. It is easy to see that (16) and (17) are satisfied when the pre-multiplication is done with $D M^{\prime}$ instead of $M^{\prime}$. The disadvantage of having to premultiply by $D$ is that after the deflation of two complex conjugate eigenpairs, the resulting $H^{\prime}$ and $U^{\prime}$ might still be complex. 


\section{References}

[1] J. R. Bunch, C. P. Nielsen, and D. C. Sorensen. Rank-one modification of the symmetric eigenproblem. Numer. Math., 31:31-48, 1978.

[2] P. A. Businger. Numerically stable deflation of Hessenberg and symmetric tridiagonal matrices. BIT, 11:262-270, 1971.

[3] J. Cuppen. A divide and conquer method for the symmetric tridiagonal eigenproblem. Numer. Math., 36:177-195, 1981.

[4] J. Dongarra. Improving the accuracy of computed matrix eigenvalues. Technical Report ANL-80-84, Argonne National Lab., August 1980.

[5] J. Dongarra, C. Moler, and J. Wilkinson. Improving the accuracy of computed eigenvalues and eigenvectors. SIAM J. Numer. Aral., 20:23-45, 1982.

[6] J. Dongarra and D. Sorensen. A fully parallel algorithm for the symmetric eigenvalue problem. SIAM J. Sci. Statist. Comput, 8:s139-s154, 1987.

[7] J. Dongarra, D. Sorensen, K. Connolly, and J. Patterson. Programming methodology and performance issues for advanced computer architectures. Parallel Computing, 8:41-58, 1988.

[8] G. Golub and J.H. Wilkinson. Ill-conditioned eigensystems and the computation of the jordan canonical form. SIAM Review, 18:578-619, 1976.

[9] G. H. Golub. Some modified matrix eigenvalue problems. SIAM Rev., 15:318-334, 1973.

[10] Gene Golub and Charles Van Loan. Matrix Computations. Johns Hopkins University Press, Baltimore, MD, second edition, 1989.

[11] Konrad Knopp. Theory of Functions. Dover Publications, New York, NY, 1945. 
[12] T.Y. Li, Zhonggang Zeng, and Luan Cong. Solving eigenvalue problems of real nonsymmetric matrices with real homotopies. Preprint, Michigan State University, E. Lansing, MI 48824-1027, 1990.

[13] G. Peters and J.H. Wilkinson. Inverse iteration, ill-conditioned equations and Newton's method. SIAM Review, 21:339-360, 1979.

[14] B. T. Smith, J. M. Boyle, J. J. Dongarra, B. S. Garbow, Y. Ikebe, V. C. Klema, and C. B. Moler. Matrix Eigensystem Routines - EISPACK Guide, volume 6 of Lecture Notes in Computer Science. Springer-Verlag, Berlin, 1976.

[15] J. Wilkinson and C. Reinsch. Handbook for Automatic Computation: Volume II - Linear Algebra. Springer-Verlag, New York, 1971.

[16] J. H. Wilkinson. The Algebraic Eigenvalue Problem. Oxford University Press, 1965. 
ORNL/TM-12003

\section{INTERNAL DISTRIBUTION}

$\begin{aligned} 1 . & \text { B. R. Appleton } \\ 2-3 . & \text { T. S. Darland } \\ 4 . & \text { E. F. D' Azevedo } \\ 5 . & \text { J. M. Donato } \\ 6-10 . & \text { J. J. Dongarra } \\ 11 . & \text { T. H. Dunigan } \\ 12 . & \text { G. A. Geist } \\ 13 . & \text { M. R. Leuze } \\ 14 . & \text { E. G. Ng } \\ 15 . & \text { C. E. Oliver } \\ 16 . & \text { B. W. Peyton } \\ 17-21 . & \text { S. A. Raby } \\ 22 . & \text { C. H. Romine }\end{aligned}$

\author{
23. T. H. Rowan \\ 24-28. R. F. Sincovec \\ 29-33. R. C. Ward \\ 34. P. H. Worley \\ 35. A. Zucker \\ 36. Central Research Library \\ 37. ORNL Patent Office \\ 38. K-25 Applied \\ Technology Library \\ 39. Y-12 Technical Library \\ 40. Laboratory Records - RC \\ 41-42. Laboratory Records Department
}

\section{EXTERNAL DISTRIBUTION}

43. Cleve Ashcraft, Boeing Computer Services, P.O. Box 24346, M/S 7L-21, Seattle, WA 98124-0346

44. Robert G. Babb, Department of Computer Science and Engineering, Oregon Graduate Institute, 19600 N.W. Walker Rd., Beaverton, OR 97006

45. David H. Bailey, NASA Ames Research Center, Mail Stop 258-5, Moffett Field, CA 94035

46. Jesse L. Barlow, Department of Computer Science, Pennsylvania State University, University Park, PA 16802

47. Edward H. Barsis, Computer Science and Mathematics, P. O. Box 5800, Sandia National Laboratories, Albuquerque, NM 87185

48. Eric Barszcz, NASA Ames Research Center, MS T045-1, Moffett Field, CA 94035

49. Robert E. Benner, Parallel Processing Division 1413, Sandia National Laboratories, P. O. Box 5800, Albuquerque, NM 87185

50. Donna Bergmark, Comell Theory Center, Engineering and Theory Center Building, Ithaca, NY 14853-3901

51. Chris Bischof, Mathematics and Computer Science Division, Argonne National Laboratory, 9700 South Cass Ave., Argonne, IL 60439

52. Ake Bjorck, Department of Mathematics, Linkoping University, S-581 83 Linkoping, Sweden 
53. Jean R. S. Blair, Department of Computer Science, Ayres Hall, University of Tennessee, Knoxville, TN 37996-1301

54. Daniel Boley, Department of Computer Science, University of Minnesota, 200 Union St. S.E. Rm.4-192 Minneapolis, MN 55455

55. Roger W. Brockett (EPMD Advisory Committee), Wang Professor of Electrical Engineering and Computer Science, Division of Applied Sciences, Harvard University, Cambridge, MA 02138

56. James C. Browne, Department of Computer Sciences, University of Texas, Austin, TX 78712

57. Bill L. Buzbee, Scientific Computing Division, National Center for Atmospheric Research, P.O. Box 3000, Boulder, CO 80307

58. Donald A. Calahan, Department of Electrical and Computer Engineering, University of Michigan, Ann Arbor, MI 48109

59. John Cavallini, Office of Scientific Computing, Office of Energy Research, ER-7, Germantown Building, U.S. Department of Energy, Washington, DC 20545

60. Ian Cavers, Department of Computer Science, University of British Columbia, Vancouver, British Columbia V6T 1W5, Canada

61. Tony Chan, Department of Mathematics, University of California, Los Angeles, 405 Hilgard Ave., Los Angeles, CA 90024

62. Jagdish Chandra, Army Research Office, P.O. Box 12211, Research Triangle Park, NC 27709

63. Eleanor Chu, Department of Computer Science, University of Waterloo, Waterloo, Ontario, Canada N2L 3G1

64. Melvyn Ciment, National Science Foundation, 1800 G Street N.W., Washington, DC 20550

65. Thomas Coleman, Department of Computer Science, Comell University, Ithaca, NY 14853

66. Paul Concus, Mathematics and Computing, Lawrence Berkeley Laboratory, Berkeley, CA 94720

67. Jane K. Cullum, IBM T. J. Watson Research Center, P.O. Box 218, Yorktown Heights, NY 10598

68. George Cybenko, Center for Supercomputing Research and Development, University of Ilinois, 104 S. Wright St., Urbana, IL 61801-2932

69. George J. Davis, Department of Mathematics, Georgia State University, Atlanta, GA 30303

70. John J. Dorning, (EPMD Advisory Committee), Department of Nuclear Engineering Physics, Thomton Hall, McCormack Rd., University of Virginia, Charlottesville, VA 22901 
71. Iain S Duff, Atlas Centre, Rutherford Appleton Laboratory, Chilton, Oxon OX11 OQX England

72. Patricia Eberlein, Department of Computer Science, SUNY at Buffalo, Buffalo, NY 14260

73. Stanley Eisenstat, Department of Computer Science, Yale University, P.O. Box 2158 Yale Station, New Haven, CT 06520

74. Lars Elden, Department of Mathematics, Linkoping University, 58183 Linkoping, Sweden

75. Howard C. Elman, Computer Science Department, University of Maryland, College Park, MD 20742

76. Albert M. Erisman, Boeing Computer Services, P.O. Box 24346, M/S 7L-21, Seattle, WA $98124-0346$

77. Ian Foster, Mathematics and Computer Science Division, Argonne National Laboratory, 9700 South Cass Ave., Argonne, IL 60439

78. Geoffrey C. Fox, Booth Computing Center 158-79, Califomia Institute of Technology, Pasadena, CA 91125

79. Paul O. Frederickson, NASA Ames Research Center, RIACS, M/S T045-1 Moffett Field, CA 94035

80. Fred N. Fritsch, Computing \& Mathematics Research Division, Lawrence Livermore National Laboratory, P. O. Box 808, L-316 Livermore, CA 94550

81. Robert E. Funderlic, Department of Computer Science, North Carolina State University, Raleigh, NC 27650

82. Dennis B. Gannon, Computer Science Department, Indiana University, Bloomington, IN 47405

83. David M. Gay, Bell Laboratories, 600 Mountain Ave., Murray Hill, NJ 07974

84. C. William Gear, Computer Science Department, University of Illinois, Urbana, IL 61801

85. W. Morven Gentleman, Division of Electrical Engineering, National Research Council, Building M-50, Room 344, Montreal Rd., Ottawa, Ontario, Canada K1A 0R8

86. J. Alan George, Vice President, Academic and Provost, Needles Hall, University of Waterloo, Waterloo, Ontario, Canada N2L 3G1

87. John R. Gilbert, Xerox Palo Alto Research Center, 3333 Coyote Hill Rd., Palo Alto, CA 94304

88. Gene H. Golub, Department of Computer Science, Stanford University, Stanford, CA 94305

89. Joseph F. Grcar, Division 8331, Sandia National Laboratories, Livermore, CA 94550

90. Sven Hammarling, Numerical Algorithms Group Ltd. Wilkinson House, Jordan Hill Road Oxford OX2 8DR, United Kingdom 
91. Per Christian Hansen, UNI*C Lyngby, Building 305. Technical University of Denmark, DK-2800 Lyngby, Denmark

92. Richard Hanson, IMSL Inc., 2500 Park West Tower One, 2500 City West Blvd., Houston, TX 77042-3020

93. Michael T. Heath, National Center for Supercomputing Applications, 4157 Beckman Institute, University of Illinois, 405 North Mathews Avenue, Urbana, IL 61801-2300

94. Don E. Heller, Physics and Computer Science Department, Shell Development Co., P.O. Box 481, Houston, TX 77001

95. Nicholas J. Higham, Department of Mathematics, University of Manchester, Grt Manchester, M13 9PL, England

96. Charles J. Holland, Air Force Office of Scientific Research, Building 410, Bolling Air Force Base, Washington, DC 20332

97. Robert E. Huddleston, Computation Department, Lawrence Livermore National Laboratory, P.O. Box 808, Livermore, CA 94550

98. Ilse Ipsen, Department of Computer Science, Yale University, P.O. Box 2158 Yale Station, New Haven, CT 06520

99. Elizabeth Jessup, University of Colorado, Department of Computer Science, Boulder, CO 80309-0430

100. Lennart Johnsson, Thinking Machines Inc., 245 First St., Cambridge, MA 021421214

101. Harry Jordan, Department of Electrical and Computer Engineering, University of Colorado, Bouider, CO 80309

102. Bo Kagstrom, Institute of Information Processing, University of Umea, 5-901 87 Umea, Sweden

103. Malvin H. Kalos, Comell Theory Center, Engineering and Theory Center Building, Comell University, Ithaca, NY 14853-3901

104. Hans Kaper, Mathematics and Computer Science Division, Argonne National Laboratory, 9700 South Cass Ave., Argonne, IL 60439

105. Robert J. Kee, Applied Mathematics Division 8331, Sandia National Laboratories, Livermore, CA 94550

106. Kenneth Kennedy, Department of Computer Science, Rice University, P.O. Box 1892, Houston, TX 77005

107. Thomas Kitchens, Department of Energy, Scientific Computing Staff, Office of Energy Research, ER-7, Office G-236 Germantown, Washington, DC 20585

108. Richard Lau, Code $1111 \mathrm{MA}, 800 \mathrm{~N}$. Quincy Street, Boston Tower, 1 Arlington, VA 22217-5000

109. Alan J. Laub, Department of Electrical and Computer Engineering, University of Califomia, Santa Barbara, CA 93106 
110. Robert L. Launer, Army Research Office, P.O. Box 12211, Research Triangle Park, North Carolina 27709

111. Charles Lawson, MS 301490, Jet Propulsion Laboratory, 4800 Oak Grove Dr., Pasadena, CA 91109

112. Peter D. Lax, Courant Institute of Mathematical Sciences, New York University, 251 Mercer St., New York, NY 10012

113. James E. Leiss (EPMD Advisory Committee), Rt. 2, Box 142C, Broadway, VA 22815

114. John G. Lewis, Boeing Computer Services, P.O. Box 24346, M/S 7L-21, Seattle, WA 98124-0346

115. Jing Li, IMSL Inc., 2500 Park West Tower One, 2500 City West Blvd., Houston, TX $77042-3020$

116. Joseph Liu, Department of Computer Science, York University, 4700 Keele St., North York, Ontario, Canada M3J 1P3

117. Franklin Luk, School of Electrical Engineering, Comell University, Ithaca, NY 14853

118. Thomas A. Manteuffel, Department oi Mathematics, University of Colorado Denver, Denver, CO 80202

119. Peter Mayes, NAG Ltd., Wilkinson House, Jordan Hill Road, Oxford, OX2 8DR, United Kingdom

112. Paul C. Messina, Mail Code 158-79, Califomia Institute of Technology, 1201 E. California Blvd. Pasadena, CA 91125

113. James McGraw, Lawrence Livermore National Laboratory, L-306, P.O. Box 808, Livermore, CA 94550

114. Cleve Moler, The Mathworks, 325 Linfield Place, Menlo Park, CA 94025

115. Neville Moray (EPMD Advisory Committee), Department of Mechanical and Industrial Engineering, University of Illinois, 1206 West Green Street, Urbana, IL 61801

116. Brent Morris, National Security Agency, Ft. George G. Meade, MD 20755

117. Dianne P. O'Leary, Computer Science Department, University of Maryland, College Park, MD 20742

118. James M. Ortega, Department of Applied Mathematics, Thomton Hall University of Virginia, Charlottesville, VA 22903

119. Chris Paige, Department of Computer Science, McGill University, 805 Sherbrooke St. W., Montreal, Quebec, Canada H3A 2K6

120. Roy P. Pargas, Department of Computer Science, Clemson University, Clemson, SC 29634-1906

121. Beresford N. Parlett, Department of Mathematics, University of Califorria, Berkeley, CA 94720 
122. Merrell Patrick, Department of Computer Science, Duke University, Durham, NC 27706

123. Robert J. Plemmons, Departments of Mathematics and Computer Science, North Carolina State University, Raleigh, NC 27650

124. Jesse Poore, Department of Computer Science, Ayres Hall, University of Tennessee, Knoxville, TN 37996-1301

125. Alex Pothen, Department of Computer Science, Pennsylvania State University, University Park, PA 16802

126. Michael J. Quinn, Computer Science Department, Oregon State University, Corvallis, OR 97331

127. Giuseppe Radicati di Brozolo, IBM European Center for Scientific and Engineering Computing, 00147 Roma, via Giorgione 159, Italy

128. Noah Rhee, Department of Mathematics, University of Missouri-Kansas City, Kansas City, MO 64110-2499

129. John K. Reid, Numerical Analysis Group, Central Computing Department, Atlas Centre, Rutherford Appleton Laboratory, Didcot, Oxon OX11 0QX, England

130. Wemer C. Rheinboldt, Department of Mathematics and Statistics, University of Pittsburgh, Pittsburgh, PA 15260

131. John R. Rice, Computer Science Department, Purdue University, West Lafayette, IN 47907

132. Garry Rodrigue, Numerical Mathematics Group, Lawrence Livermore Laboratory, Livermore, CA 94550

133. Donald J. Rose, Department of Computer Science, Duke University, Durham, NC 27706

134. Ahmed H. Sameh, Computer Science Department, University of Illinois, Urbana, IL 61801

135. Michael Saunders, Systems Optimization Laboratory, Operations Research Department, Stanford University, Stanford, CA 94305

136. Robert Schreiber, RIACS, Mail Stop 230-5, NASA Ames Research Center, Moffet Field, CA 94035

137. Martin H. Schultz, Department of Computer Science, Yale University, P.O. Box 2158 Yale Station, New Haven, CT 06520

138. David S. Scott, Intel Scientific Computers, 15201 N.W. Greenbrier Pkwy., Beaverton, OR 97006

139. Lawrence F. Shampine, Mathematics Department, Southern Methodist University, Dallas, TX 75275

140-145. Majed Sidani, Computer Science Department, University of Tennessee. Knoxville. TN 37996 
146. Kermit Sigmon, Department of Mathematics, University of Florida, Gainesville, FL 32611

147. Horst Simon, Mail Stop 258-5, NASA Ames Research Center, Moffett Field, CA 94035

148. Larry Snyder, Department of Computer Science and Engineering, FR-35, University of Washington, Seattle, WA 98195

149. Danny C. Sorensen, Department of Mathematical Sciences, Rice University, P. O. Box 1892, Houston, TX 77251

150. Rick Stevens, Mathematics and Computer Science Division, Argonne National Laboratory, 9700 South Cass Ave., Argonne, Il 60439

151. G. W. Stewart, Computer Science Department, University of Maryland, College Park, MD 20742

152. Quentin F. Stout, Department of Electrical and Computer Engineering, University of Michigan, Ann Arbor, MI 48109

153. Daniel B. Szyld, Department of Computer Science, Duke University, Durham, NC 27706-2591

154. W.-P. Tang, Department of Computer Science, University of Waterloo, Waterloo, Ontario, Canada N2L 3G1

155. Michael Thomason, Department of Computer Science, Ayres Hall, University of Tennessee, Knoxville, TN 37996-1301

156. Bernard Tourancheau, LIP ENS-Lyon 69364 Lyon cedex 07, France

157. Charles Van Loan, Department of Computer Science, Comell University, Ithaca, NY 14853

158. James M. Varah, Centre for Integrated Computer Systems Research, University of British Columbia, Office 2053-2324 Míain Mall, Vancouver, British Columbia V6T 1W5, Canada

159. Robert G. Voigt, ICASE, MS 132-C, NASA Langley Research Center, Hampton, VA 23665

160. Michael Vose, Department of Computer Science, Ayres Hall, University of Tennessee, Knoxville, TN 37996-1301

161. Phuong Vu, Cray Research Inc., 1408 Northland Dr., Mendota Heights, MN 55120

162. E. L. Wachspress, Department of Mathematics, University of Tennessee, Knoxville, TN 37996-1300

163. Daniel D. Warner, Department of Mathematical Sciences, O-104 Martin Hall, Clemson University, Clemson, SC 29631

164. D. S. Watkins, Department of Pure and Applied Mathematics, Washington State University, Pullman, WA 99164-2930 
165. Mary F. Wheeler (EPMD Advisory Committee), Rice University, Department of Mathematical Sciences, P.O. Box 1892, Houston, TX 77251

166. Andrew B. White, Computing Division, Los Alamos National Laboratory, Los Alamos, NM 87545

167. Michael Wolfe, Oregon Graduate Institute, 19600 N.W. von Neumann Dr., Beaverton, OR 97006

168. Margaret Wright, Bell Laboratories, 600 Mountain Ave., Murray Hill, NJ 07974

169. David Young, University of Texas, Center for Numerical Analysis, RLM 13.150, Austin, TX 78731

170. Office of Assistant Manager for Energy Research and Development, U.S. Department to Energy, Oak Ridge Operations Office, P.O. Box 2001, Oak Ridge, TN 37831-8600

171-180. Office of Scientific Technical Information, P.O. Box 62, Oak Ridge, TN 37831 

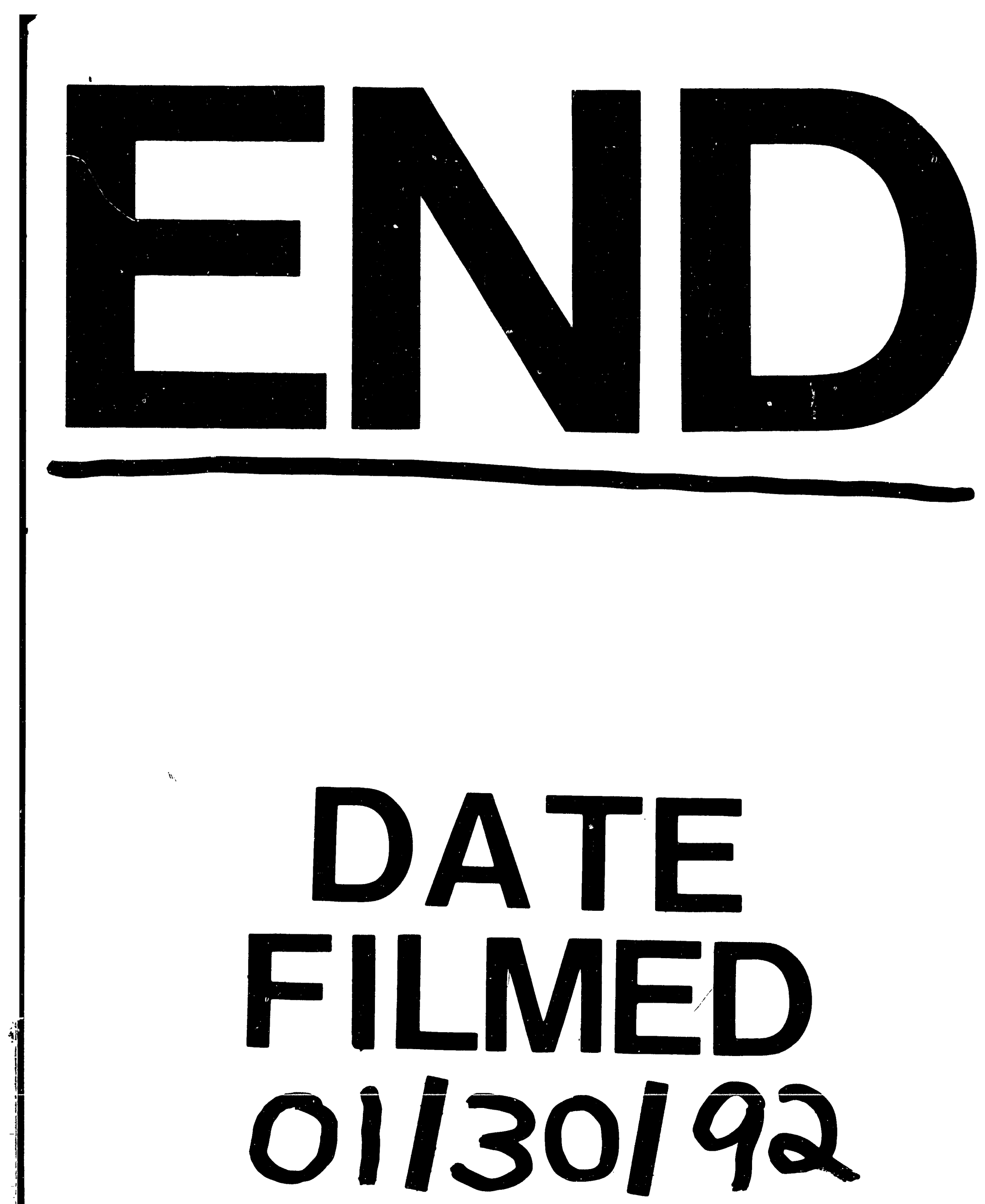


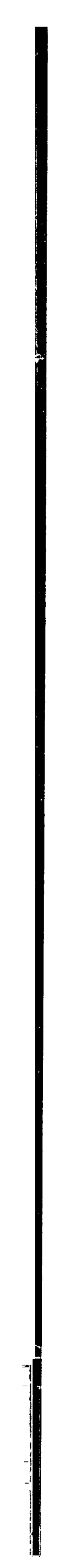

